\title{
Étude expérimentale et analyse théorique de l'anisotropie induite du sable d'Hostun
}

\author{
Induced anisotropy in Hostun sand : \\ experiments and theoretical analysis
}

\author{
J. LANIER \\ Institut de Mécanique de Grenoble* \\ C. DI PRISCO, R. NOVA \\ Politecnico di Milano**
}

Rev. Franç. Géotech. n 57 , pp. $59-74$ (octobre 1991)

\section{Résumé}

L'anisotropie initiale et induite est une caractéristique importante de la structure des matériaux granulaires. Dans le cas du sable d'Hostun que nous étudions ici, l'anisotropie initiale paraît faible alors que les effets de l'anisotropie induite sont très importants. Nous les mettons en évidence dans cet article pour différents types de sollicitations: essais isotropes, essais radiaux à pression moyenne constante, essais axisymétriques linéaires ou à volume constant. On montre en particulier que l'anisotropie induite peut être une cause de liquéfaction même pour des sables denses.

Nous notons également que la plupart des effets observés peuvent être interprétés dans le cadre de l'élastoplasticité avec écrouissage cinématique.

\section{Abstract}

Inherent and induced anisotropy is a matter of fact in granular materials. In this paper we study experimental behavior of Hostun sand. We point out the very important effects of induced anisotropy created by a first initial loading. This anisotropy is identified on different paths like isotropic or deviatoric test, axisymmetric linear or isochoric tests. For this last case, we show that liquefaction can occur even with dense samples.

We notice that many of these effects can be explain by elastoplastic modelling.

\footnotetext{
- Domaine universitaire, B.P. $\mathrm{n}^{\circ} 53$ X, 38041 Grenoble Cedex.

* Viale Sabotino 19/2, 20135 Milan, Italie.
} 


\section{INTRODUCTION}

L'anisotropie induite a souvent été évoquée pour expliquer le comportement fortement irréversible des géomatériaux. D'un point de vue microstructural, la création d'une structure anisotrope due au chemin de déformation a été mise en évidence grâce à l'étude de la "fabrique " des échantillons de sable (ODA, 1972). D'un point de vue macroscopique, c'est seulement depuis la fin des années 70 (ARTHUR et al., 1977, 1980, 1987 ; YAMADA, ISHIHARA, 1979 ; LANIER, 1983) qu'on a pu développer des études expérimentales grâce à des moyens d'essais complexes (cellule de cisaillement DSC, presse 3D, cylindre creux).

Les effets de l'anisotropie se sont alors révélés d'une très grande importance, surtout pour ce qui concerne la modification de la rigidité du matériau selon les différentes directions de chargement. La prise en compte de ces effets dans la modélisation devenait de ce fait nécessaire pour avoir une approche réaliste du comportement des géomatériaux.

Nous avons développé un programme expérimental visant à mettre en évidence, d'un point de vue macroscopique, les effets dus aux changements de structure du matériau créés par différents chemins de chargements initiaux. Cette étude expérimentale a été développée sur la presse 3D de l'Institut de Mécanique de Grenoble. Avec cet appareillage il est possible d'étudier le comportement du matériau dans le plan bissecteur $\left(\sigma_{2}=\sigma_{3}\right)$ et dans le plan déviatoire, les axes principaux de l'essai restant confondus avec les axes de la machine. Il n'y a pas la possibilité de tester les effets de rotation continue comme on peut le faire avec le DSC ou le cylindre creux (SYMES et al., 1984). Une description de la presse utilisée et certains résultats concernant l'anisotropie des sables denses ont déjà été présentés (CAMBOU, LANIER, 1988). Cet article a pour but de compléter et de développer les résultats dans ce domaine. Notre programme expérimental comprend trois parties :

1. Etude de l'anisotropie induite créée par une première charge de compression sur un sable très lâche. La mise en évidence de l'anisotropie est obtenue par comparaison de chemins radiaux dans un plan déviatoire $(b=0,1,0,5)$.

2. Effet de l'anisotropie induite sur des chemins axisymétriques (sable dense) pour deux types de chemin : - chemin à volume constant ;

- chemin linéaire dans le plan $(\mathrm{p}, \mathrm{q})$.

3. Chemins axisymétriques $\sigma_{1} / \sigma_{3}=$ constante: recherche expérimentale d'un domaine de réversibilité créé par une première charge (sable dense).

Dans la suite on utilisera les notations suivantes :

$\mathrm{S} 1=\operatorname{tr}(\sigma)=3 \cdot \mathrm{p}$

$\mathrm{SD} 2=\sqrt{\operatorname{tr}\left(\mathrm{s}^{2}\right)}$, où $\mathrm{s}$ est le déviateur de contrain-

te. Dans les conditions axisymétriques,

$$
\begin{array}{r}
\operatorname{SD} 2=\sqrt{2 / 3}\left|\sigma_{1}-\sigma_{3}\right|=|\mathrm{Q}| . \\
\epsilon_{\mathrm{v}}=\operatorname{Ln}(\mathrm{V} / \mathrm{Vo}) \text { et } \epsilon_{\mathrm{i}}=-\operatorname{Ln}\left(\mathrm{X}_{\mathrm{i}} / \mathrm{X}_{\mathrm{io}}\right)
\end{array}
$$

$\mathrm{ID} 2=\sqrt{\operatorname{tr}\left(\mathrm{e}^{2}\right)}$, où e est le déviateur de déformation; dans les conditions axisymétriques,

$$
\operatorname{ID} 2=\sqrt{2 / 3}\left|\epsilon_{1}-\epsilon_{3}\right|=|\Gamma| \text {. }
$$

Les contraintes et déformations de compression sont comptées positives.

$\mathrm{X}, \mathrm{Y}, \mathrm{Z}$ sont les axes de la presse, $\mathrm{Z}$ étant la direction verticale.

\section{INFLUENCE DE LA MÉTHODE DE PRÉPARATION DES ÉCHANTILLONS SUR LES RÉSULTATS EXPÉRIMENTAUX}

Le sable utilisé est le sable fin d'Hostun (RF) dont on trouvera une description complète dans FLAVIGNY et al. (1990).

Les échantillons sont préparés denses ou lâches : pour obtenir une forte densité nous utilisons la méthode de damage par couches, à sec. Les échantillons lâches sont préparés légèrement humides $(3 \%$ de teneur en eau) ; cette faible humidité confère au sable une structure très dispersée qui se maintient même après la compression isotrope. Nous avons obtenu pour les échantillons dense des densités de l'ordre de $16 \mathrm{kN} / \mathrm{m}^{3}$, et pour les échantillons lâches $13 \mathrm{kN} / \mathrm{m}^{3}$. Ce mode de préparation fournit des échantillons qui peuvent présenter une faible anisotropie : la figure 1 représente les chemins de déformation déviatoire suivis pour 2 essais de compression classique (sable lâche), l'un sur l'axe vertical $Z$, l'autre sur l'axe horizontal X. On note pour ce dernier que $\epsilon_{\mathrm{y}} \# \epsilon_{\mathrm{z}}$, ce qui peut être interprété comme une anisotropie initiale du matériau. De même, la figura $2 \mathrm{a}$ présente les résultats d'un essai isotrope (sable dense) pour lequel les trois déformations $\epsilon_{\mathrm{x}}, \epsilon_{\mathrm{y}}, \epsilon_{\mathrm{z}}$ ne sont pas égales.

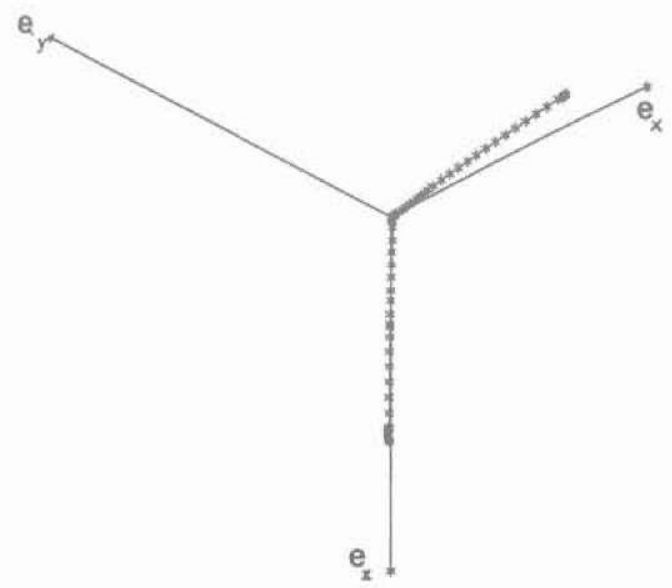

\section{PLAN DEVIATOIRE DE DEFORMATION}

Fig. 1. - Chemins de déformation déviatoires pour deux essais de compression sur les directions $Z$ et $X$ (échantillons vierges). Fig. 1. - Deviatoric strain paths for two compression tests on $X$ and $Z$ directions (virgin samples). 

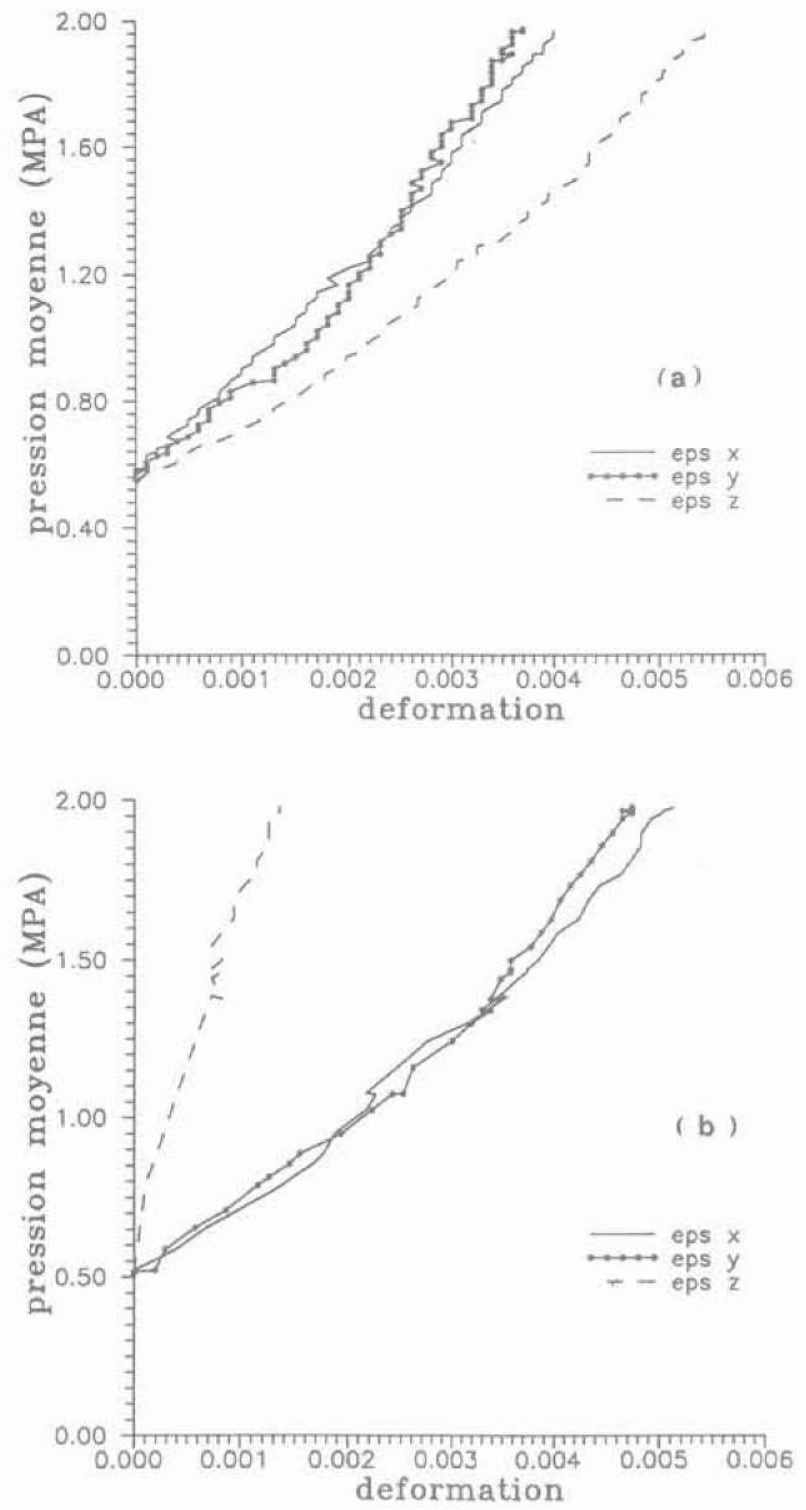

Fig. 2. - Déformations $\epsilon_{\mathrm{X}}, \epsilon_{\mathrm{y}}, \epsilon_{\mathrm{z}}$ pour un essai isotrope (sable dense) : (a) échantillon vierge,

(b) après une compression sur la direction Z.

Fig. 2. - Isotropic tests on dense sand: evolution of $\epsilon_{\mathrm{x}}, \epsilon_{\mathrm{y}}, \epsilon_{\mathrm{z}}$ versus $p$ : (a) virgin sample,

(b) after a compression test

on the Z-direction.

Ces effets d'anisotropie initiale sont faibles. Ils sont d'autre part sujet à une dispersion due à la non répétabilité des conditions expérimentales (méthode de préparation, mise en place des échantillons, conditions de mesures...). Les effets d'anisotropie induite que nous nous proposons d'étudier sont au contraire très reproductibles et traduisent réellement l'effet d'une première charge. La figure $2 \mathrm{~b}$ présente un essai isotrope réalisé après une compression dans la direction $Z$. L'anisotropie induite par cette première charge se manifeste par un «durcissement * de la direction $Z$ $\left(\epsilon_{\mathrm{z}}<\epsilon_{\mathrm{x}}=\epsilon_{\mathrm{y}}\right)$.

Afin d'évaluer la dispersion de nos mesures et par conséquent de fixer dans quelle mesure on peut don- ner un sens rhéologique à nos comparaisons de résultats, nous donnons sur les figures $3 a, b, c, d$ les courbes de contrainte et de variation de volume pour plusieurs essais répétés, théoriquement identiques. La figure $3 a-b$ correspond à un essai de compression classique sur un sable dense, la figure $3 c-3 d$ à un essai de compression à pression moyenne constante sur sable lâche. La répétabilité n'est pas parfaite. Les écarts observés nous donnent un ordre de grandeur de la précision que nous pouvons attendre. Toute tentative d'interprétation rhéologique d'effets inférieurs à ces écarts serait dénuée de sens.

\section{RÉSULTATS EXPÉRIMENTAUX POUR CHEMINS RADIAUX DANS UN PLAN DÉVIATOIRE (SABLE LÂCHE)}

Pour mettre en évidence expérimentalement le comportement anisotrope d'un matériau on doit comparer des essais ayant la même histoire de contraintes principales mais orientés différemment par rapport aux axes matériels de l'échantillon. Notre presse permet seulement de réaliser des permutations des directions principales majeure, mineure et intermédiaire, c'està-dire des rotations de $90^{\circ}$. La méthode que nous avons utilisée est la suivante:

a. à partir d'un échantillon isotrope (ou quasiment isotrope comme nous venons de le voir), on réalise une première charge de compression sur Z $\left(\sigma_{\mathrm{x}}=\sigma_{\mathrm{y}}\right.$ $=0,5 \mathrm{MPa}$ ) jusqu'à $25 \%$ pour une première série d'essais, et $10 \%$ pour une deuxième. Puis par décharge, on revient à l'état de contrainte isotrope initial. Cette première histoire crée l'anisotropie que l'on se propose d'étudier ;

b. on réalise ensuite un chemin radial dans le plan déviatoire $(p=0,5 \mathrm{MPa})$. Sept essais sont ainsi réalisés parmi lequels on peut comparer : deux essais de compression $(b=0)$, deux essais d'extension $(b=$ 1) et trois essais $b=0,5$ (fig. 4).

Rappelons que le coefficient b est défini par:

$$
\mathrm{b}=\left(\sigma_{2}-\sigma_{3}\right) /\left(\sigma_{1}-\sigma_{3}\right) \text { avec } \sigma_{1}>\sigma_{2}>\sigma_{3}
$$

Nous utiliserons également l'angle de Lode $\alpha_{\sigma}$ qui repère l'orientation des chemins radiaux dans le plan déviatoire et que l'on peut définir par:

$$
\operatorname{tg} \alpha_{o}=\sqrt{3} \cdot \mathrm{b} /(2-\mathrm{b})
$$

Ces essais ont été effectués avec des échantillons de sable très lâches initialement $\left(\gamma=13 \mathrm{kN} / \mathrm{m}^{3}\right)$. Après la première charge de compression le matériau reste lâche $\left(\gamma=14 \mathrm{kN} / \mathrm{m}^{3}\right)$.

Les résultats obtenus sont présentés sur les figures $5 \mathrm{a}$ à $5 \mathrm{f}$ pour la première série, $6 \mathrm{a}$ à $6 \mathrm{f}$ pour la seconde.

L'importance de l'anisotropie, créée par la charge de compression, est clairement mise en évidence sur ces courbes. Comme pour les sables denses (CAMBOU, LANIER, 1988) on observe que les modules initiaux des courbes SD2/S1-ID2 diminuent lorsque le che- 

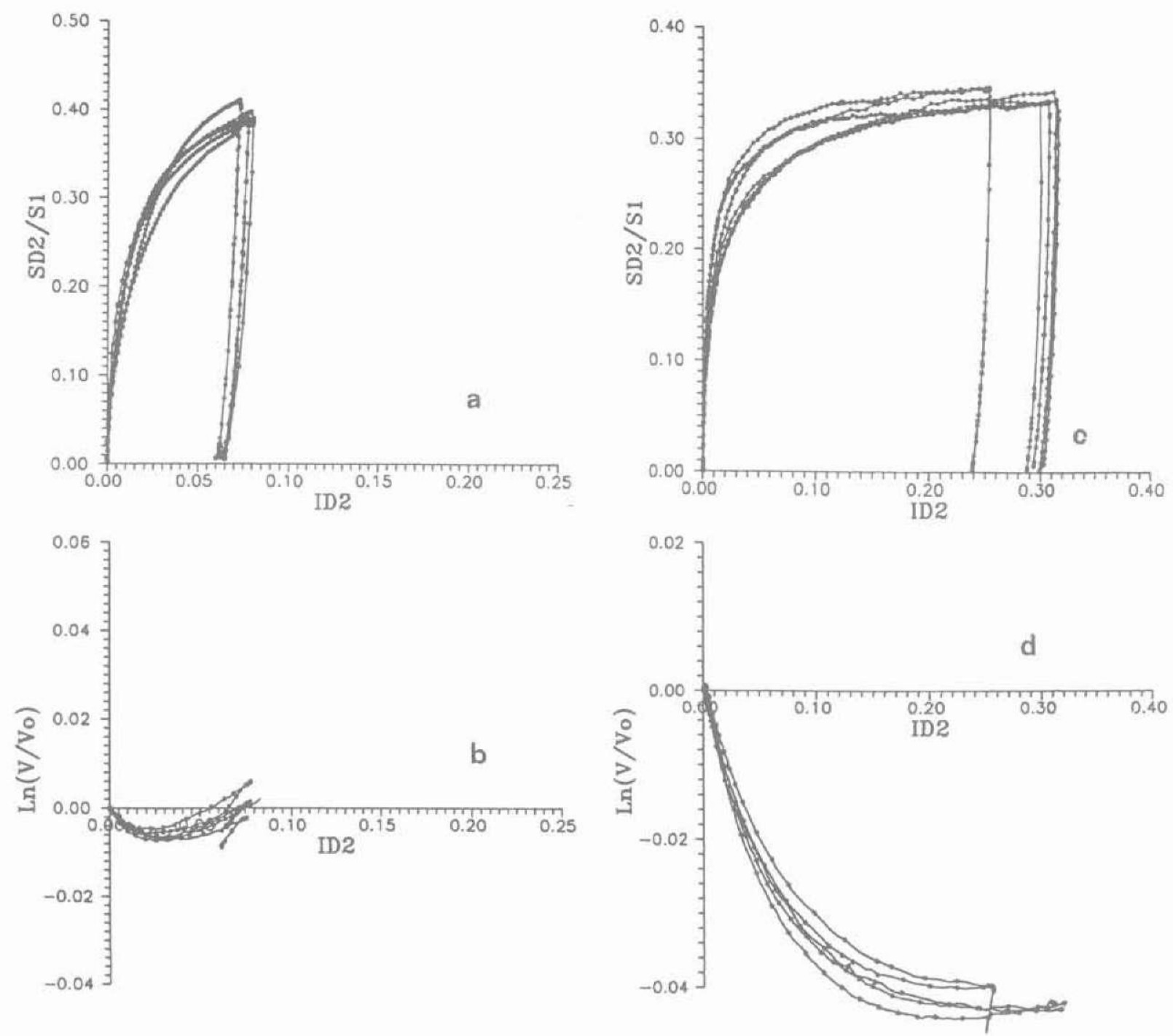

Fig. 3. - Répétabilité des essais : courbes contrainte-déformation et variation de volume:

(a) et (b) essais de compression: $\sigma_{x}=\sigma_{y}=0,5 \mathrm{MPa}$ (sable dense), (c) et (d) essais de compression : $p=0,5 \mathrm{MPa}$ (sable lâche).

Fig. 3. - Repetitiveness of compression tests (stress-strain curves and volume changes)

(a), (b) $\sigma_{x}=\sigma_{y}=0,5 \mathrm{MPa}$ (dense sand), (c), (d) $p=0,5 \mathrm{MPa}$ (loose sand).

Fig. 4. - Série d'essais radiaux : $p=0,5 \mathrm{MPa}$, $b=$ constante, réalisés pour l'étude de l'anisotropie induite.

Fig. 4. - Deviatoric tests performed

to study induced anisotropy $p=0,5 \mathrm{MPa}, b=0,1,0,5$.

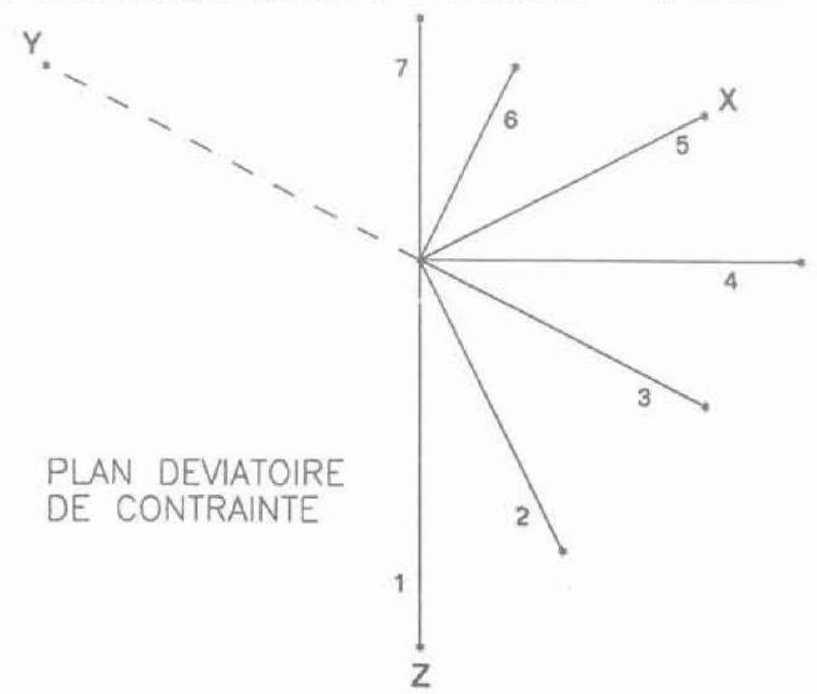

min radial " s'écarte » du chemin de première charge : il est maximum pour la recharge (compression sur direction Z), minimum pour l'extension sur Z.

Parallèlement le compactage du matériau augmente quand le module diminue. Le maximum de compactage est obtenu pour une valeur de SD2/S1 qui ne paraît dépendre que de $\mathrm{b}$. Ce résultat indique que la notion d'angle caractéristique est indépendante de l'anisotropie induite et par conséquent de l'histoire subie par le matériau.

La comparaison des figures 5 et 6 montre l'influence de la déformation subie par le matériau pendant la première charge $\left(\epsilon_{\mathrm{z}}=25 \%\right.$ et $\left.10 \%\right)$. Les allures des courbes ne sont pas très différentes, ce qui signifie qu'après un certain degré de déformation la structure interne du matériau reste la même: il n'y a plus d'évolution structurelle interne lorsque la déformation plastique lors de la première charge devient suffisamment importante. Dans le cadre d'une théorie élastoplastique, les variables d'écrouissage isotrope et cinématique doivent donc être choisies de façon à ce 

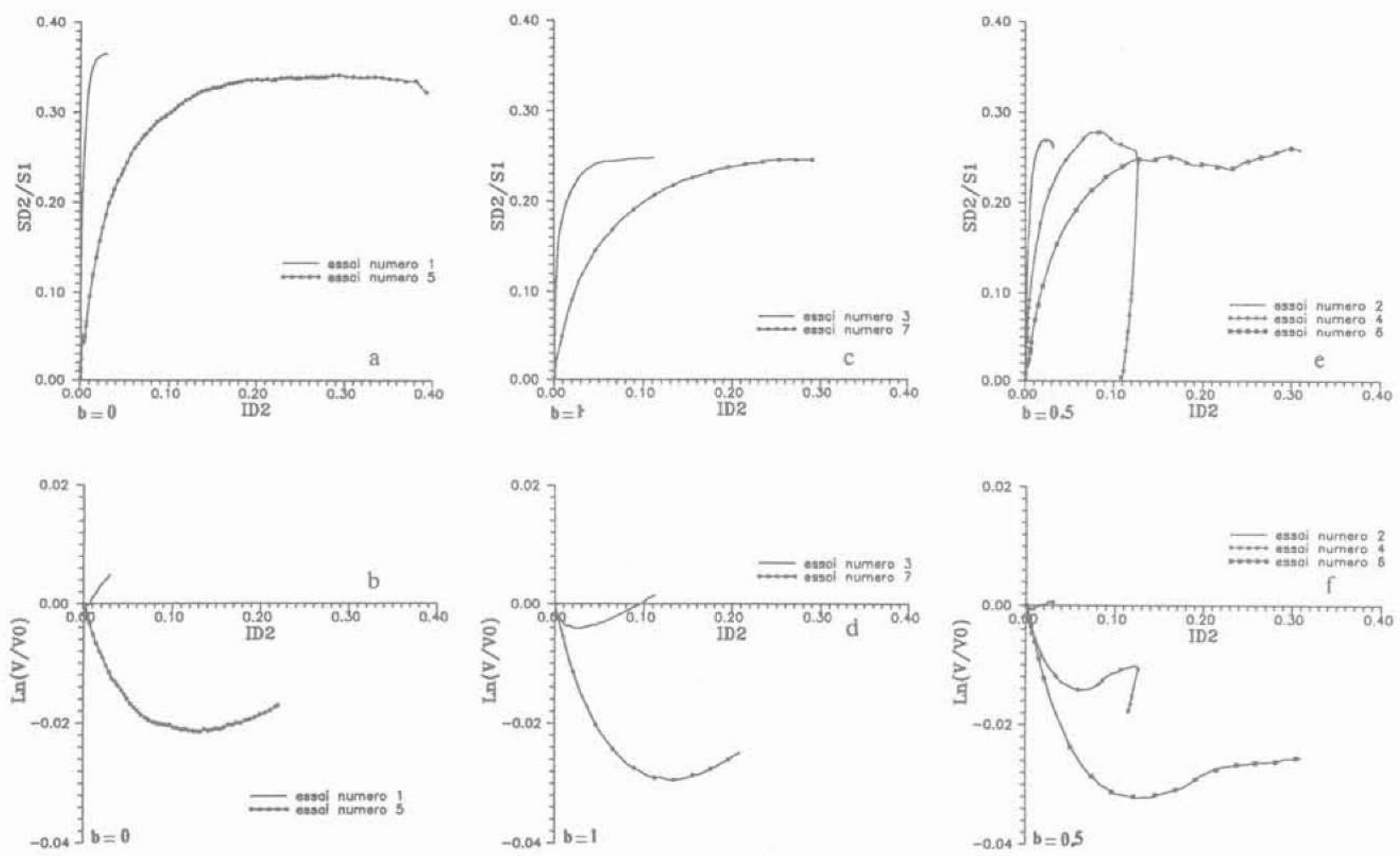

Fig. 5. - Mise en évidence de l'anisotropie induite par une compression sur la direction $Z\left(\epsilon_{\mathrm{z}}=25 \%\right)$ : comparaison des courbes contrainte-déformation et variation de volume pour une même valeur de $b$ :

(a), (b): $b=0-(c),(d): b=1-(e),(f): b=0,5$.

Fig. 5. - Induced anisotropy after a compression test $\left(\epsilon_{z}=25 \%\right)$ : comparison of deviatoric tests with the same $b$ value.
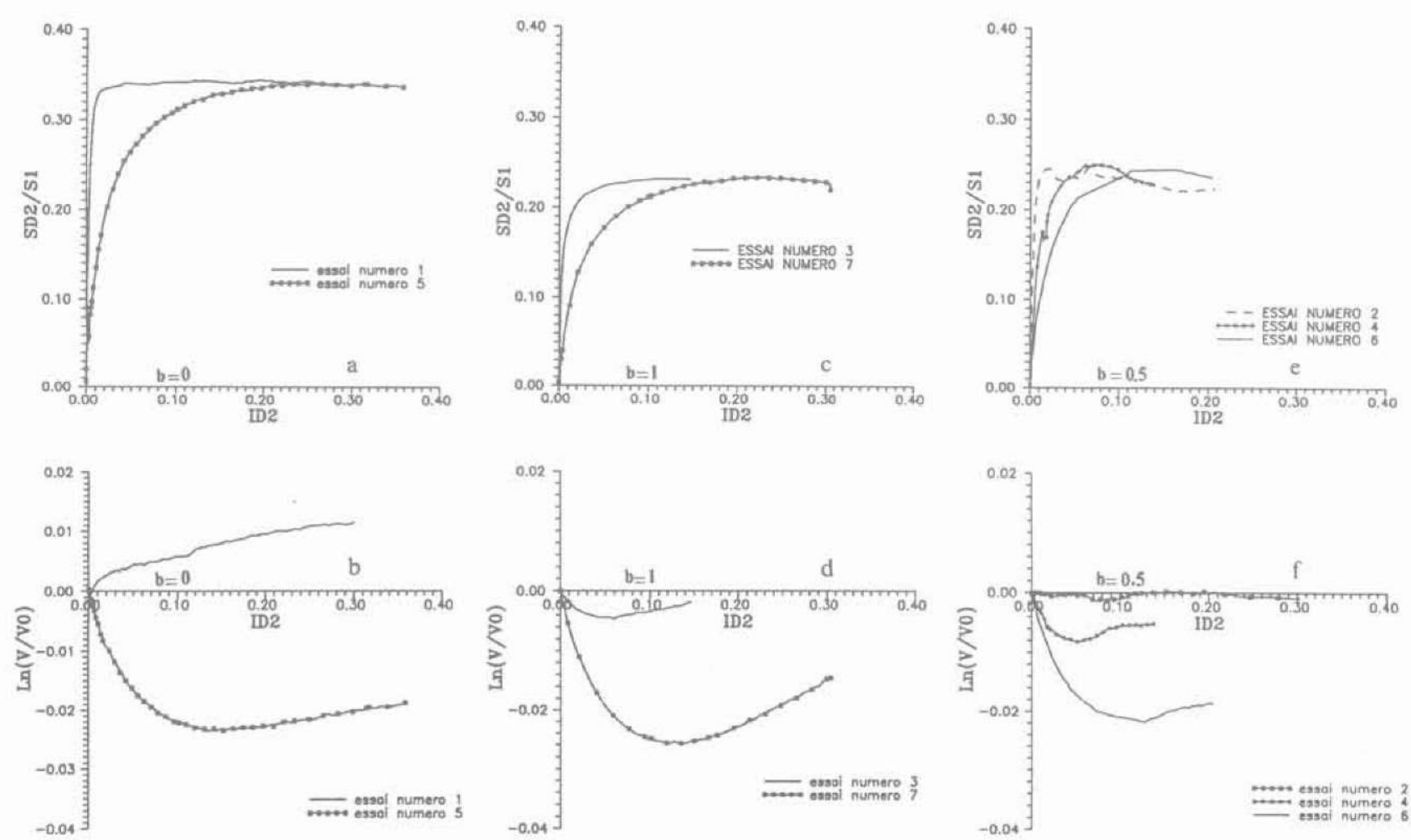

Fig. 6. - Mise en évidence de l'anisotropie induite par une compression sur la direction $Z\left(\epsilon_{z}=10 \%\right)$ : comparaison des courbes contrainte-déformation et variation de volume pour une même valeur de $b$ : (a), (b): $b=0-(c),(d): b=1-(e),(f): b=0,5$.

Fig. 6. - Induced anisotropy after a compression test $\left(\epsilon_{z}=10 \%\right)$ : comparison of deviatoric tests with the same $b$ value. 
qu'elles tendent vers une valeur limite quj correspond à l'écoulement plastique parfait du matériau, sans modification de son état.

La figure 7 permet de comparer les tésultats que nous venons de commenter avec ceux obtenus précédemment sur le sable dense. Les valeurs de SD2/S1 et de la variation de volume pour ID2 $=1$ \% y sont représentées en fonction de $\alpha_{a}$. On observe les mêmes allures pour les deux sables (dans le cas du sable dense nous avons représenté quatre séries d'essais : trois séries correspondent à une première charge de compression avec $\varepsilon_{z}=3,6,12 \%$ et une sêrie à une première charge d'extension, $\epsilon_{2}=$ - 6\%). On peut également remarquer que le
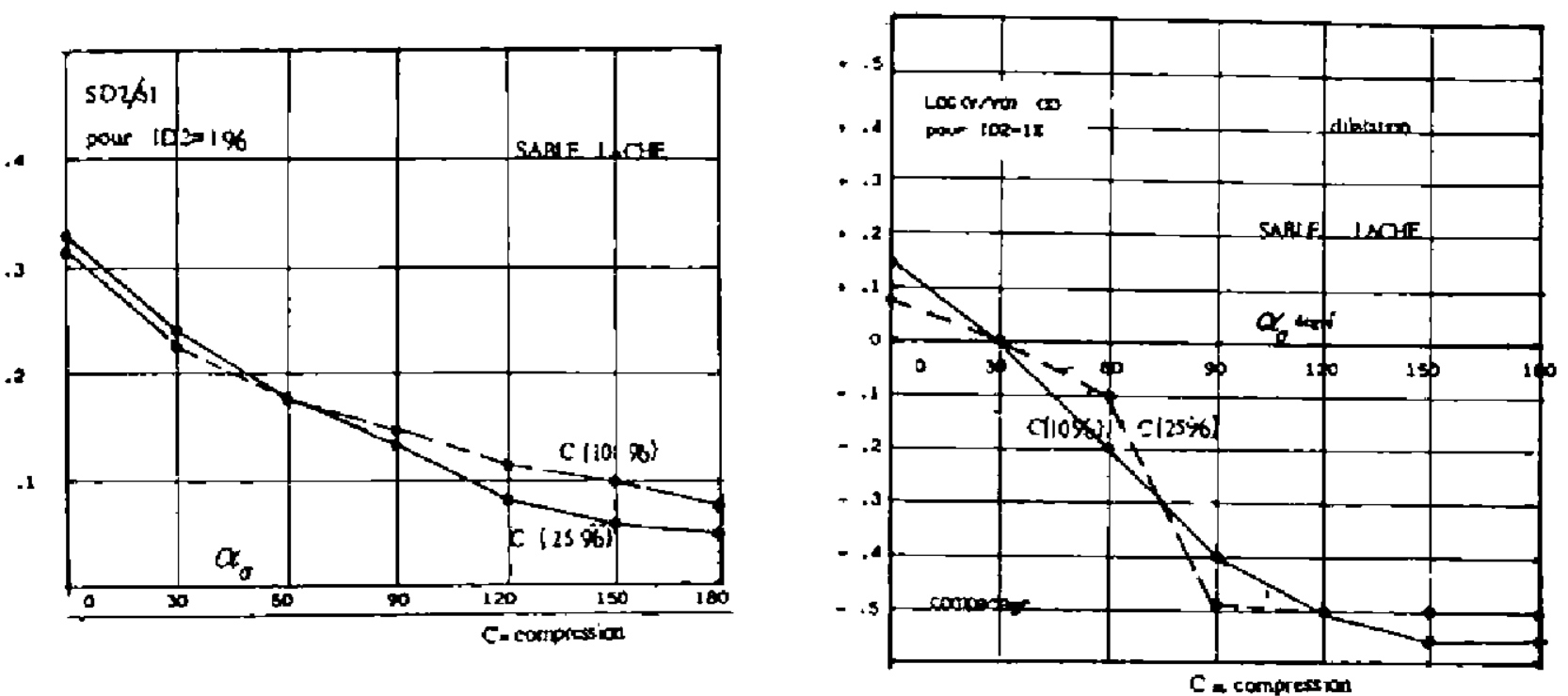

(a)
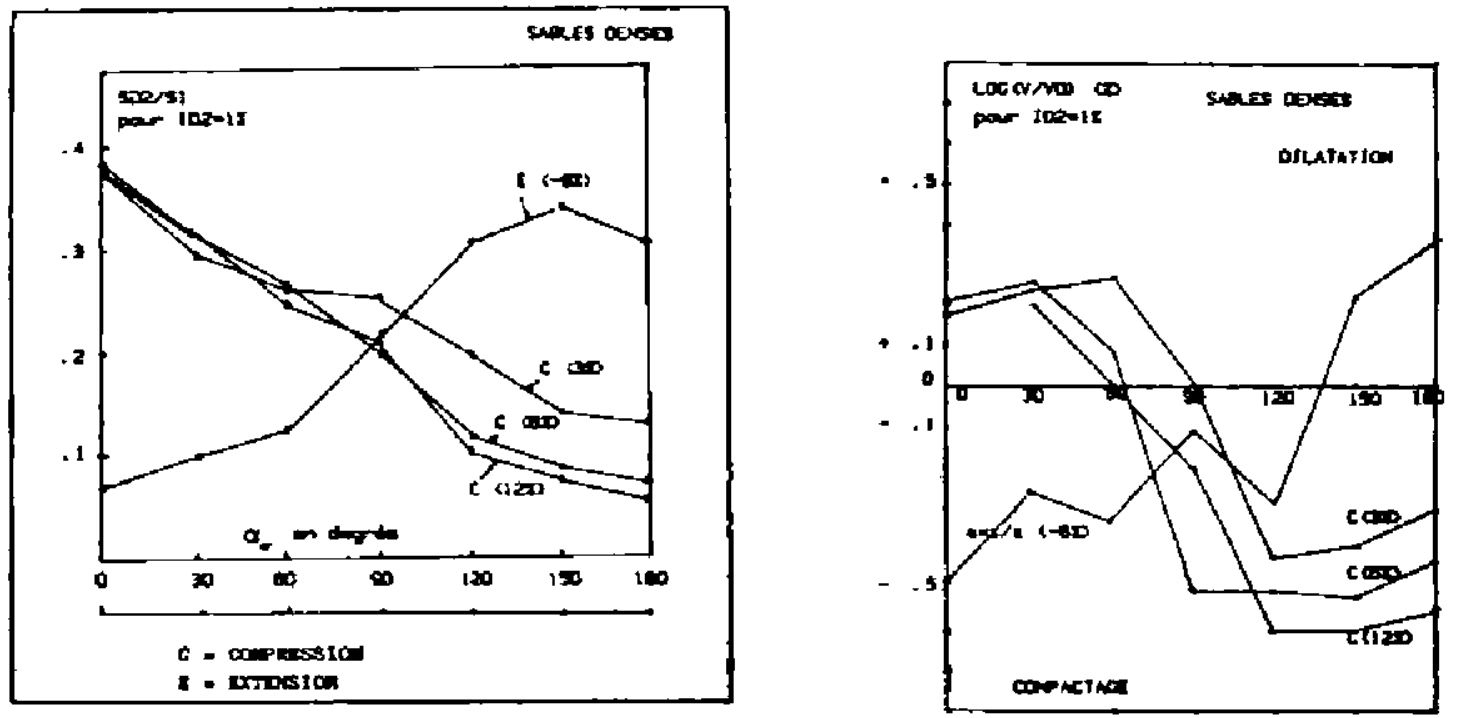

(b)

Fig. 7. - Module sécant et variation de volume pour une dóformation $102=1 \%$ en fonction de $\alpha_{g}$ : (a) sable lache. (b) sable dense.

Fig. 7. - Secant modulus and volume change for $1 D 2=1$ \% versus the loading diraction $\alpha_{\sigma}$ In $[$-plane. (a) loose sand - (b) dense sand. 
module sécant (valeur de SD2/S1 pour ID2 = $1 \%$ ) évolue régulièrement en fonction de $\alpha_{\sigma}$, alors que les variations de volume présentent une évolution rapide au voisinage de $\alpha_{\sigma}=90^{\circ}$. Ce comportement est également illustré sur la figure 8 qui regroupe les courbes de variation de volume pour les 7 essais radiaux (sable lâche); les essais $4,5,6,7$ ont pratiquement la même tangente initiale, alors que les essais 1,2 , 3 sont nettement séparés.

Pour mettre en évidence l'anisotropie, on peut également considérer la réponse cinématique des échantillons. La figure 9 représente les chemins de déformation déviatoire suivis au début de la charge radiale (petites déformations). On y constate en particulier que les essais 5 et 3 qui sont axisymétriques en contrainte ne le sont pas en déformation.

Ces résultats peuvent être qualitativement expliqués par une théorie élastoplastique avec écrouissage cinématique. En effet, si l'on suppose que le point initial du chargement (état isotrope de contrainte) appartient à une surface de charge, il peut exister des déformations plastiques si le chemin de contrainte est dirigé vers l'extérieur de cette surface dont la normale $n$, dans le plan déviatoire, a nécessairement la direction d'extension $/ Z$ (essai $n^{\circ} 7$ ) puisque la première charge admet cette direction comme axe de symétrie. Il en est nécessairement de même pour le potentiel plastique que le matériau soit standard ou non. Ainsi les déformations plastiques sont maximales pour l'essai

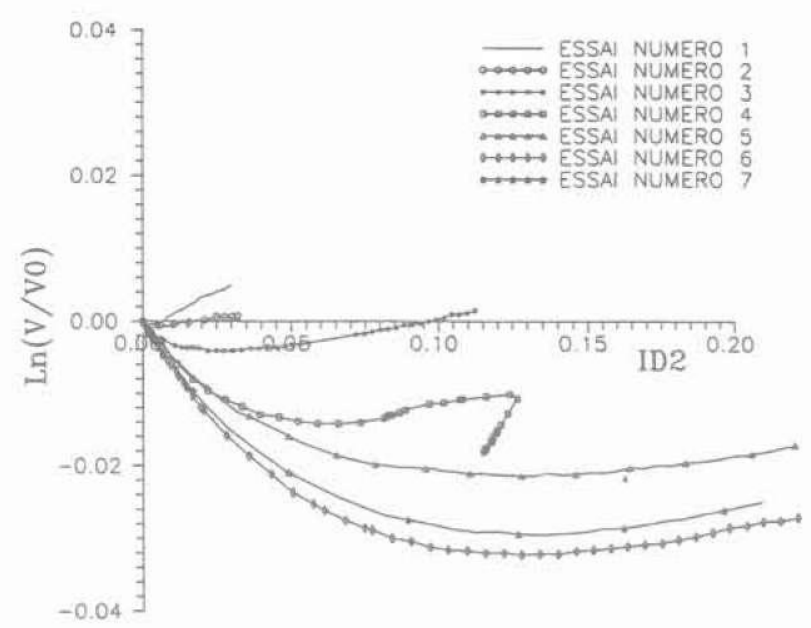

Fig. 8. - Courbe de variation de volume pour les 7 essais radiaux (après compression sur la direction $Z, \epsilon_{Z}=25 \%$ - sable lâchel. Fig. 8. - Volume change for the 7 deviatoric tests (after a compression test on the Z-direction, $\epsilon_{z}=25 \%$ - loose sand).

$\mathrm{n}^{\circ} 7$ (module le plus faible), et le chargement neutre correspond à $\alpha_{\sigma}=90^{\circ}$ (direction perpendiculaire à n). La forme de la surface de charge dans le plan déviatoire peut être imaginée par l'allure des lignes isodéformations. Les figures $10 \mathrm{a}-\mathrm{b}$ donnent l'allure de

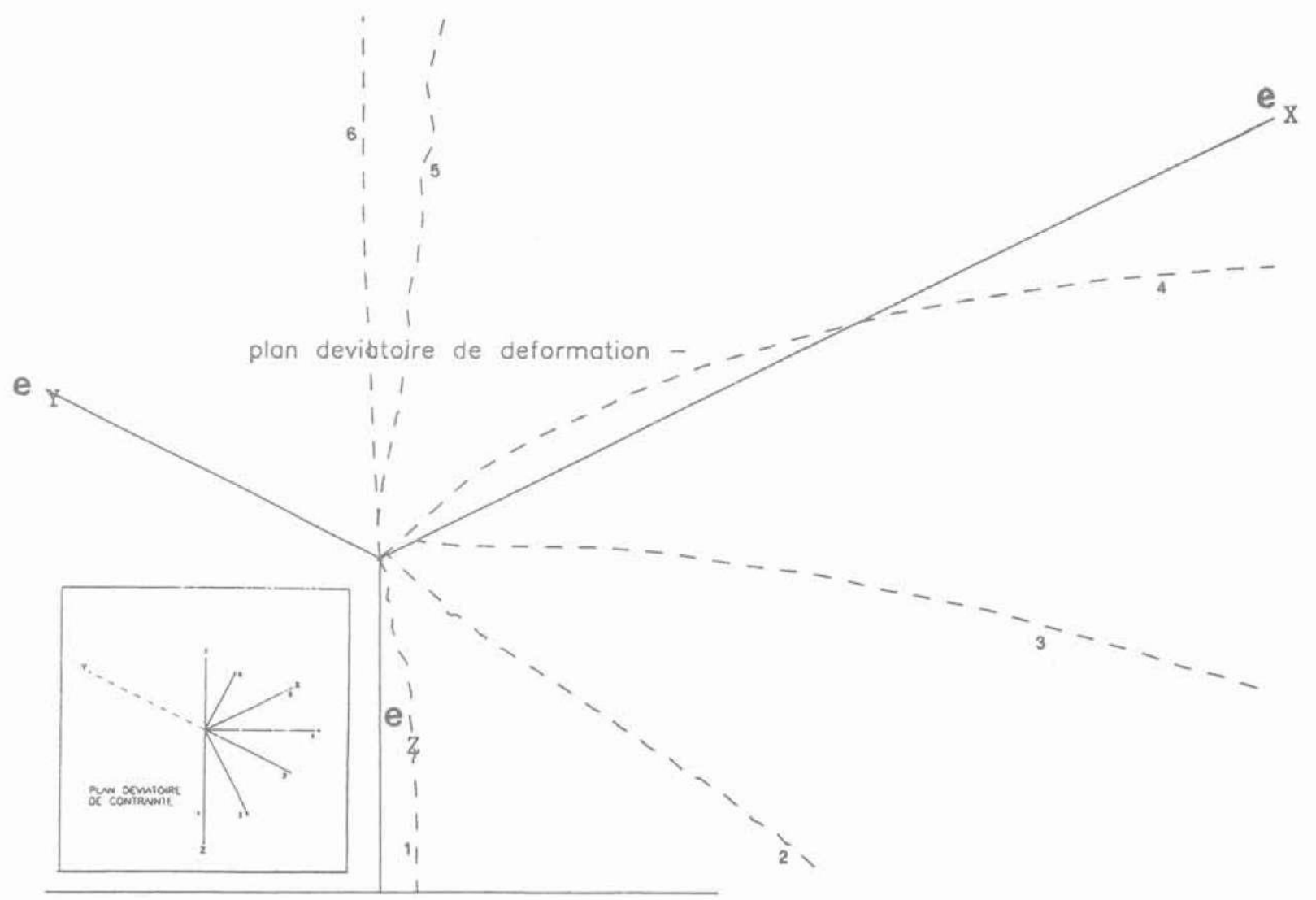

Fig. 9. - Chemins déviatoires de déformation associés aux débuts des chemins radiaux de contrainte (après compression. sur la direction $Z, \epsilon_{z}=25 \%$, sable lâche).

Fig. 9. - Deviatoric strain paths associated with deviatoric stress paths lafter a compression test on the Z-direction, $\epsilon_{z}=25 \%$, loose sand). 


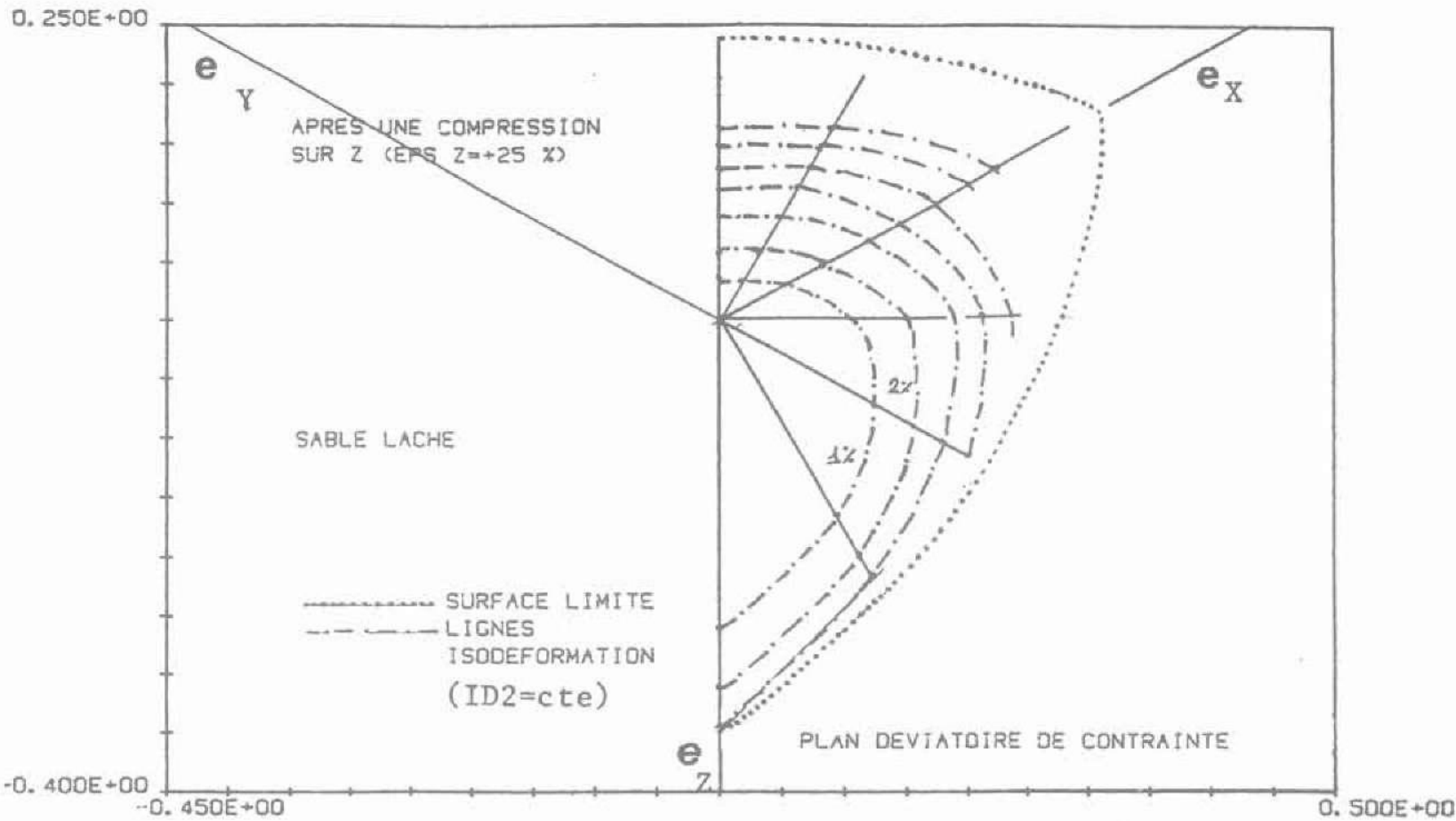

a

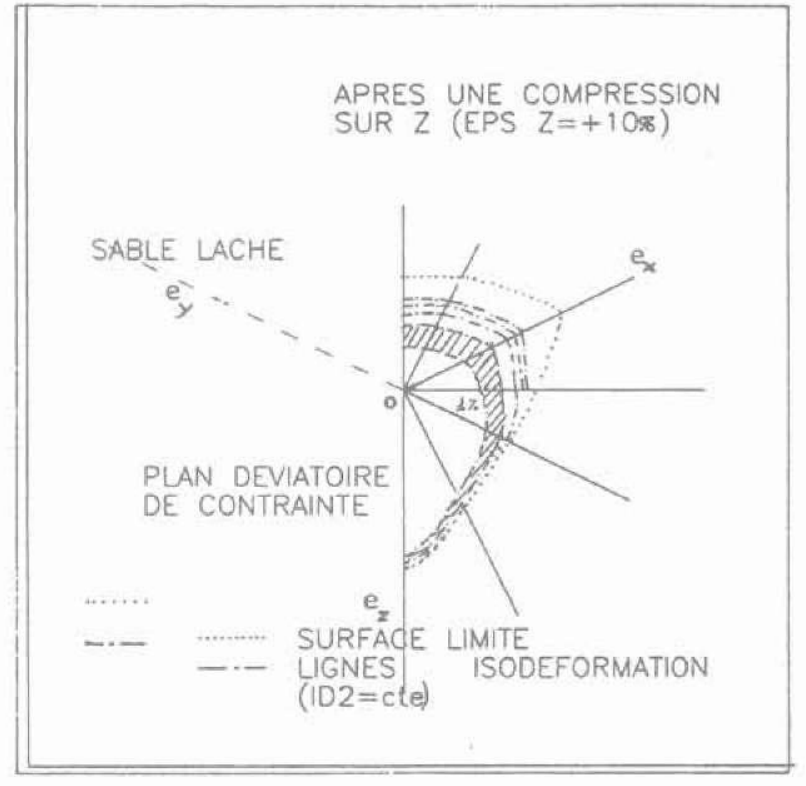

b

ces lignes pour les deux séries d'essais que nous avons effectués après compression sur la direction Z $\left(\epsilon_{2}=25 \%\right.$ et $\left.10 \%\right)$. Pour les obtenir on a reporté sur chaque chemin radial le point contrainte correspondant à ID2 $=1 \%, 2 \% \ldots$ On met alors en évidence la translation de la surface de charge dans la direction de la première charge, ce qui est caractéristique d'un écrouissage cinématique. Cette figure, purement déviatoire ne permet pas de représenter le comportement isotrope (variation de volume).
Fig. 10. - Courbes isodéformations (ID2 $=1,2, \ldots \%$ ) dans le plan déviatoire de contrainte après une compression sur la direction $Z$ (sable lâche) : (a) $\epsilon_{z}=25 \%$ - (b) $\epsilon_{z}=10 \%$. Fig. 10. - Lines of iso-deviatoric strains in II-stress plane $(I D 2=1,2, \ldots \%)$ after a compression test on Z-direction.

\section{RÉSULTATS EXPÉRIMENTAUX POUR ESSAIS AXISYMÉTRIQUES}

\subsection{Essais à volume constant (sable dense)}

Ce type d'essais, réalisé sur la presse 3D avec des échantillons secs, simule les essais non drainés communément réalisés sur les presses classiques avec des échantillons saturés. On obtient ici directement le chemin de contrainte effective sans passer par une mesure de pression interstitielle. La principale difficulté pratique est le contrôle de la variation de volume qui ne reste jamais tout à fait nulle. La figure 11 montre l'efficacité de la régulation pour un essai de charge et décharge. L'échelle qui a été choisie pour cette figure est grande. Elle correspond à l'ordre de grandeur de celle que l'on utilise pour un essai classique de compression à contrainte latérale constante * drainé ». La régulation paraît donc acceptable, mais il reste des difficultés au démarrage de l'essai où de très faibles variations de volume peuvent influencer fortement le chemin de contrainte.

Les résultats du paragraphe précédent nous ont montré que l'anisotropie induite modifie considérablement les courbes de variation de volume. On peut donc s'attendre à ce que, après une première charge de compression classique, le comportement du matériau sur les chemins à volume constant soit également 


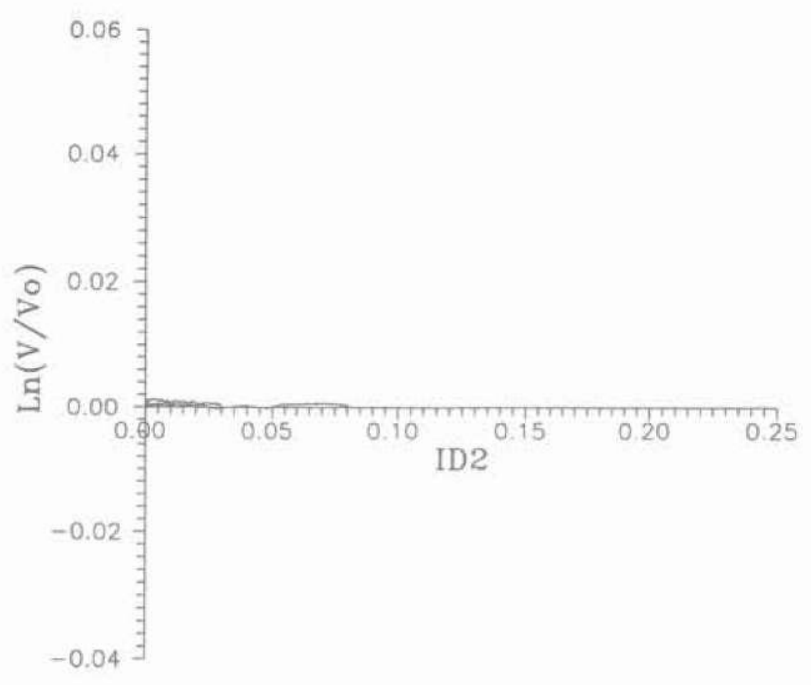

Fig. 11. - Efficacité de la régulation à " volume constant " (sable dense).

Fig. 11. - Efficienty of "constant colume" control (dense sand).

modifié : si le chemin « drainé » est compactant, la pression moyenne doit diminuer pour compenser la tendance au compactage; elle doit augmenter dans le cas contraire. La figure $12 \mathrm{a}$ donne les chemins de contrainte suivis pour trois essais à volume constant (sable dense) en extension :

- essai 1: extension sur la direction $\mathrm{Z}$ pour un échantillon vierge (matériau isotrope) ;

- essai 2 : extension sur la direction $Y$ après une compression classique "drainée "sur la direction $Z$ $\left(\epsilon_{z}=6 \%\right)$;

- essai 3 : extension sur la direction $Z$ après une compression classique * drainée * sur la direction Z $\left(\epsilon_{\mathrm{z}}=6 \%\right)$.

De la même façon la figure 13 a donne les chemins de contrainte suivis par trois essais de compression à volume constant (sable dense)

- essai 1: compression sur la direction Z pour un échantillon vierge ;

- essai 2 : compression sur la direction $Y$ après une compression classique "drainée » sur la direction $\mathrm{Z}$ $\left(\epsilon_{z}=6 \%\right)$;

- essai 3 : compression sur la direction $\mathrm{Z}$ après une compression classique "drainée "sur la direction Z $\left(\epsilon_{\mathrm{z}}=6 \%\right)$.

Les figures $12 \mathrm{~b}$ et $13 \mathrm{~b}$ donnent les courbes contraintes-déformation (SD2/S1-ID2) pour ces deux séries d'essais. Les perturbations de début d'essai que nous avons évoquées précédemment sont visibles sur les chemins de contraintes dans le plan (p-q) mais n'apparaissent pas sur ces courbes car les déformations correspondantes sont très faibles.

L'effet de la première charge, et donc de l'anisotropie induite, est ici encore très marqué. On voit en effet sur la figure $12 \mathrm{a}$ que l'essai $\mathrm{n}^{\circ} 3$ atteint presque un état de "liquéfaction " (contraintes effectives
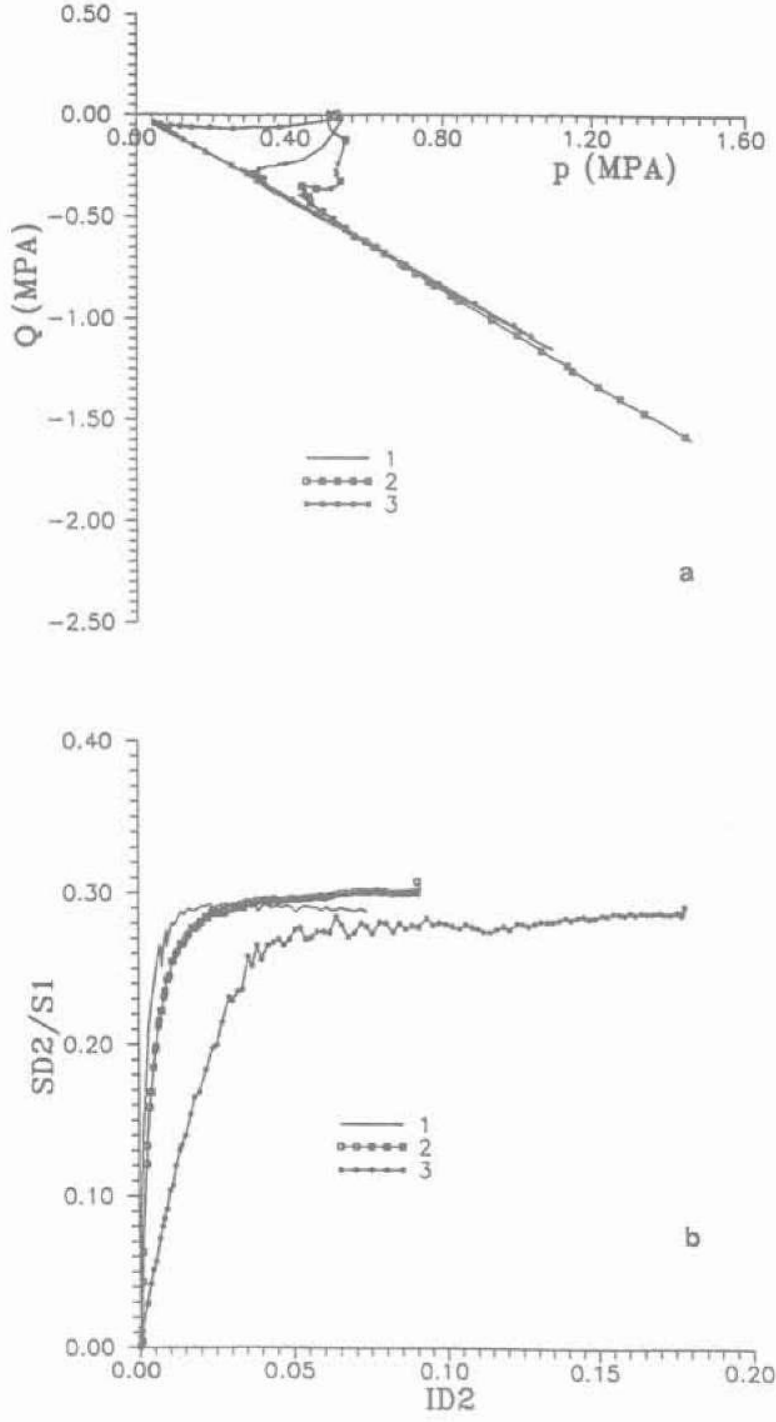

Fig. 12. - Influence de l'anisotropie induite sur les essais à volume constant en extension (sable dense).

(a) Chemins de contrainte dans le plan $p-Q$.

(b) Courbes contrainte-déformation.

Fig. 12, - Induced anisotropy effects for "constant volume" test in extension (dense sand)

(a) stress paths in ( $p$ - $Q)$ plane, (b) stress-strain curves.

nulles) avant que la pression moyenne n'augmente et que le chemin de contrainte ne "remonte " une surface limite à angle de frottement constant, le même pour les trois essais. Il est alors clair que le risque de liquéfaction ne doit pas seulement être caractérisé par une densité initiale comme on le fait dans l'étude classique de la liquéfaction, mais aussi par l'histoire précédente c'est-à-dire l'état structurel du matériau.

La figure $14 a-b$ donne les résultats d'un essai cyclique à volume constant qui confirme l'importance de la première charge. Après la première charge de compression 0-1, l'échantillon est déchargé. Le chemin de décharge 1-2-3 repasse par un état isotrope de contrainte voisin de l'état initial. La pression moyenne continue de diminuer jusqu'au point 2 (quasi liquéfaction) puis augmente jusqu'au point 3 . Une nouvelle décharge est alors entreprise 3-4-5. Là encore 

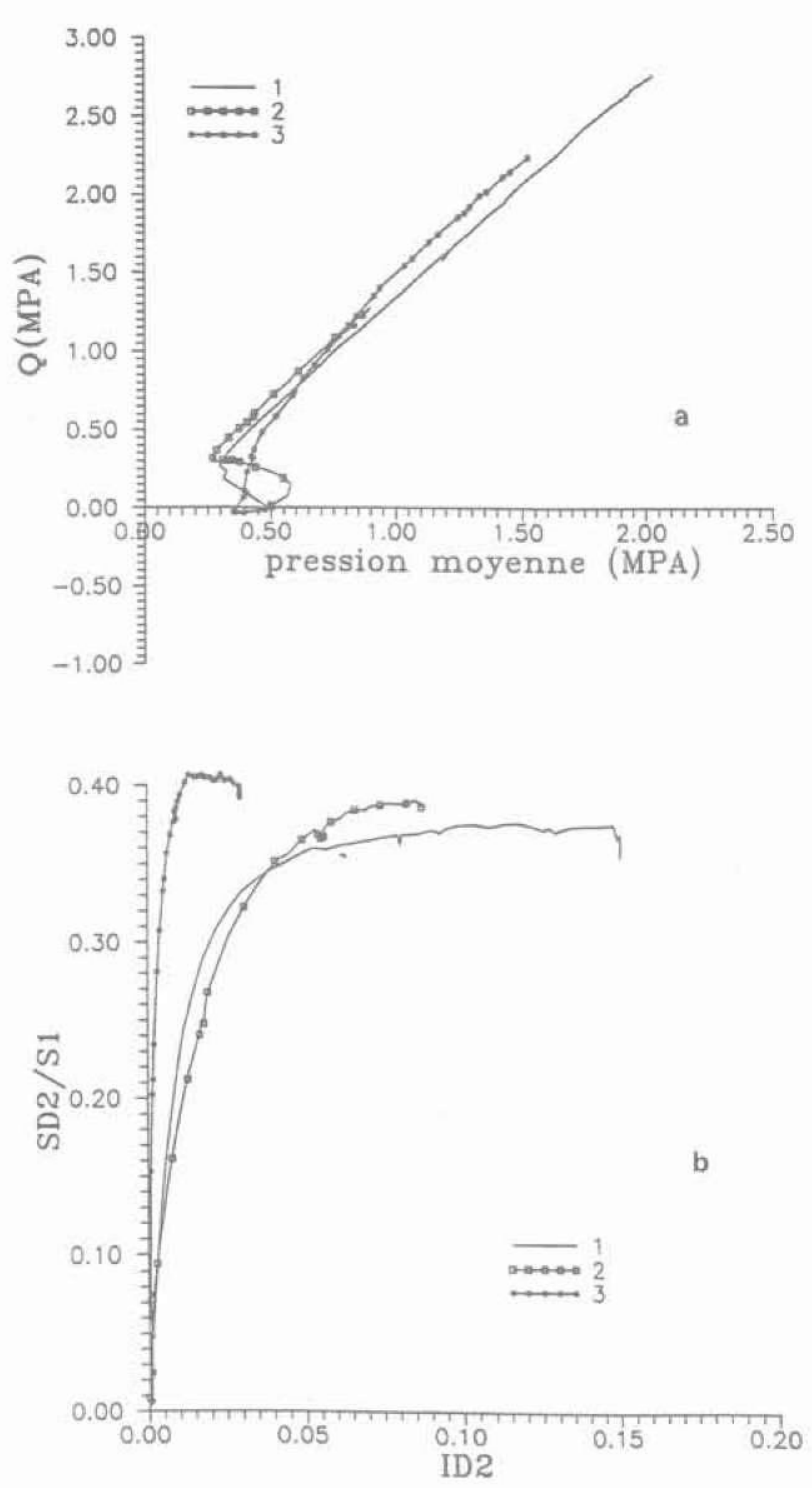

Fig. 13. - Influence de l'anisotropie induite sur les essais à volume constant en compression (sable dense).

(a) Chemins de contrainte dans le plan $p-Q$.

(b) Courbes contrainte-déformation.

Fig. 13. - Induced anisotropy effects for "constant volume " test in compression (dense sand):

(a) stress paths in (p-Q) plane, (b) stress-strain curves.

la liquéfaction est atteinte (point 4) avant que la pression moyenne n'augmente à nouveau jusqu'au point 5. La figure $14 \mathrm{~b}$ permet de suivre l'évolution du déviateur q au cours de l'essai. On notera que, pour que le sens de variation de la pression moyenne s'inverse (points 2 et 4), il est nécessaire de faire subir à l'échantillon des déformations importantes. Ce phénomène peut être interprété en disant que la structure anisotrope induite par le chargement précédent de compression (respectivement extension) rend le matériau incapable de supporter l'état de contrainte d'extension (respectivement de compression) tant que cette structure n'a pas été modifiée par une déformation suffisante.
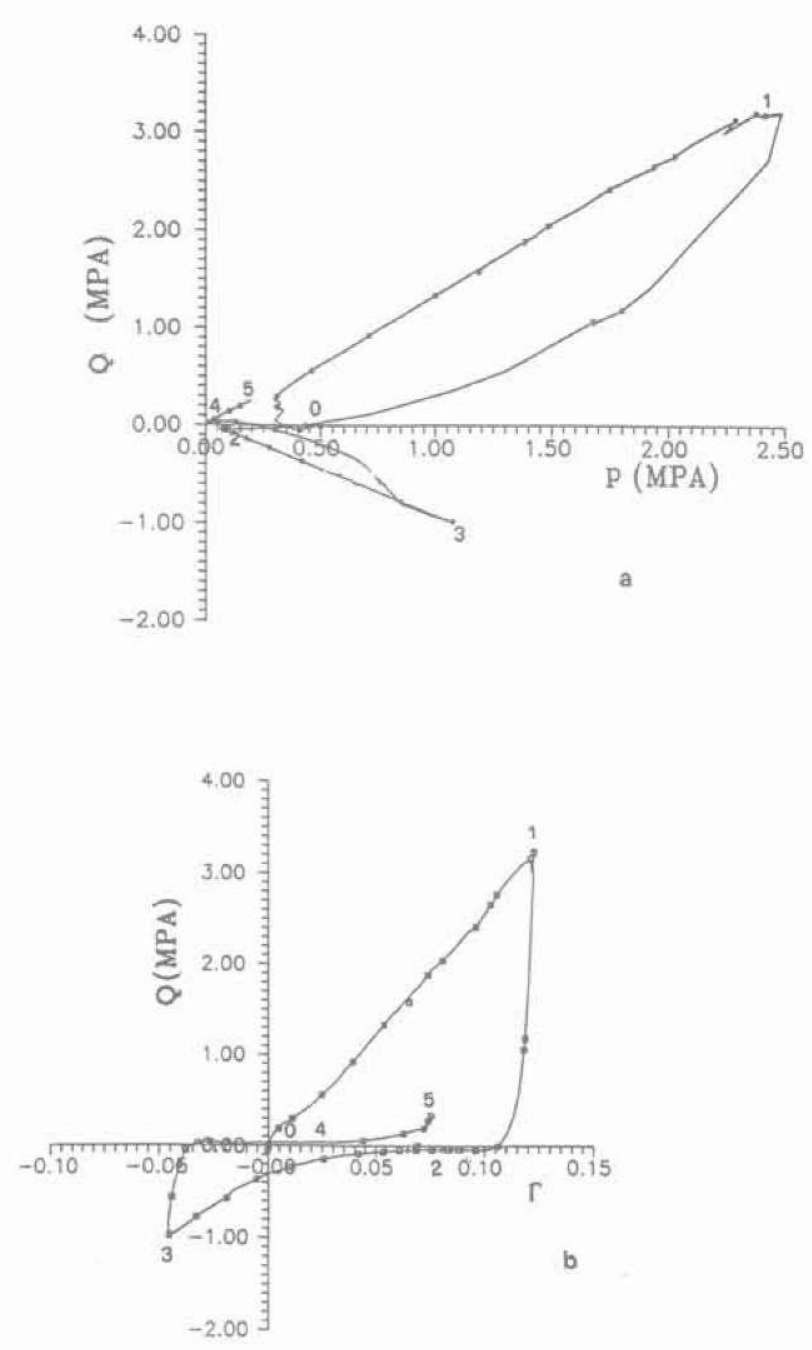

Fig. 14. - Essai cyclique de compression-extension à volume constant (sable dense) :

(a) chemin de contrainte dans le plan p-Q, (b) courbes Q- $\Gamma$.

Fig. 14. - Cyclic test of compression-extension with constant volume.

(a) stress path in $(p-Q)$ plane, (b) $Q-\Gamma$ curves.

\subsection{Chemins axisymétriques radiaux (sable dense)}

Les essais radiaux dans un plan déviatoire nous ont montré qu'une première charge de compression ou d'extension créait une forte anisotropie du matériau, Il nous a paru intéressant d'étudier le comportement du matériau anisotrope dans le plan $\mathrm{p}-\mathrm{Q}$, c'est-à-dire quand la pression moyenne varie. Nous avons pour cela réalisé trois types de chemins axisymétriques de compression ou d'extension ( 6 essais) définis par (fig. 15) :

- $\sigma_{\mathrm{x}}=\sigma_{\mathrm{y}}=0,5 \mathrm{MPa}$ (chemin triaxial classique); - $\mathrm{p}=0,5 \mathrm{MPa}$ (chemin à pression moyenne constante) ;

- $\sigma_{z}=0,5 \mathrm{MPa}$ (chemin à contrainte axiale constante) ; 


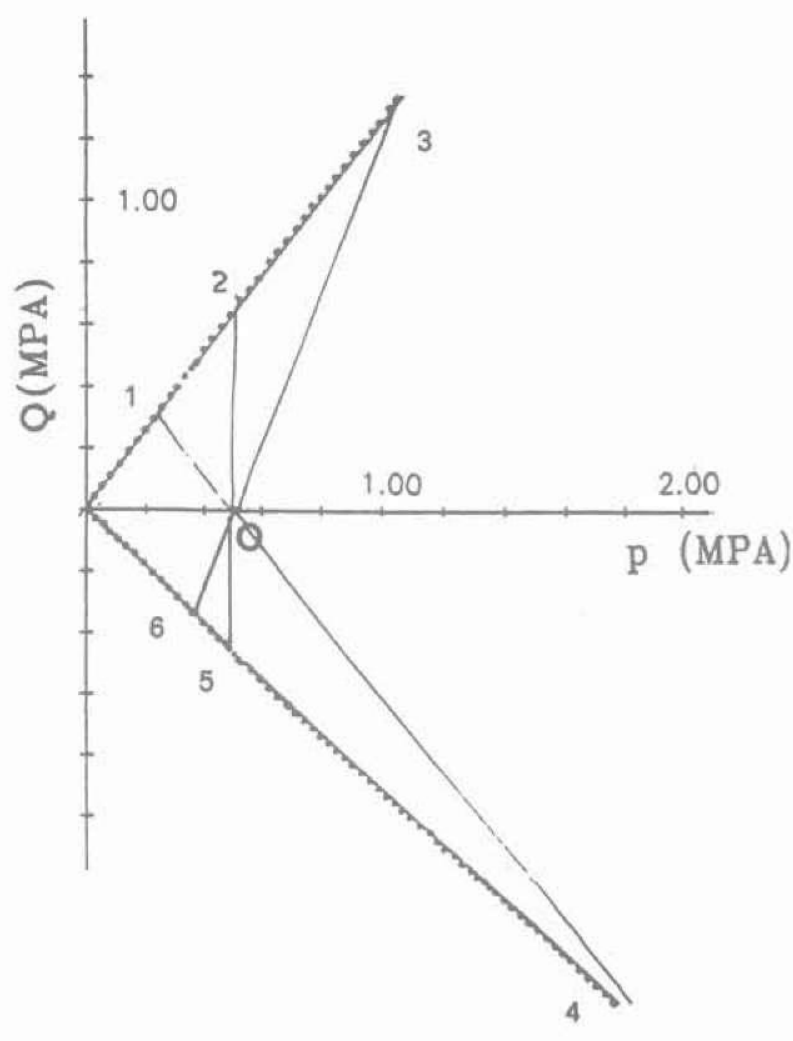

Fig. 15. - Série d'essais axisymétriques de compression et d'extension réalisés pour l'étude de l'anisotropie induite.

Fig. 15. - Axisymeric tests in compression and extension performed to study induced anisotropy.

pour lesquels la pression moyenne augmente, reste constante, ou diminue. Cette série d'essais est réalisée avec trois types d'échantillons:

- des échantillons vierges (considérés comme isotropes) ;

- des échantillons anisotropes, l'anisotropie étant créée par une première charge de compression $\left(\epsilon_{\mathrm{z}}=\right.$ $6 \%)$, ou d'extension $\left(\epsilon_{\mathrm{z}}=-5 \%\right)$.

Dans ce cas l'observation de l'anisotropie ne peut pas être directe car les résultats que nous allons comparer ne correspondent pas au même type d'échantillon. Nous mettrons simplement en évidence la différence de comportement entre nos trois types d'échantillons, cette différence étant due à l'anisotropie que nous avons pu caractériser précédemment.

Nous avons regroupé sur la figure 16a les courbes contrainte-déformation(SD2/S1-ID2) pour les essais de compression. On notera que pour chaque type d'échantillon, on peut admettre que la courbe de charge est presque indépendante du chemin suivi. Par contre, pour les échantillons qui avaient subi une première charge de compression (respectivement d'extension) la rigidité est plus forte (resp. plus faible) que celle des échantillons vierges. La résistance maximum est peu modifiée. Des remarques similaires peuvent être faites pour la figure $16 \mathrm{~b}$ qui présente les courbes des essais d'extension.

Les courbes de la figure 17 donnent les variations de volume pour les différents essais réalisés. On peut y remarquer :
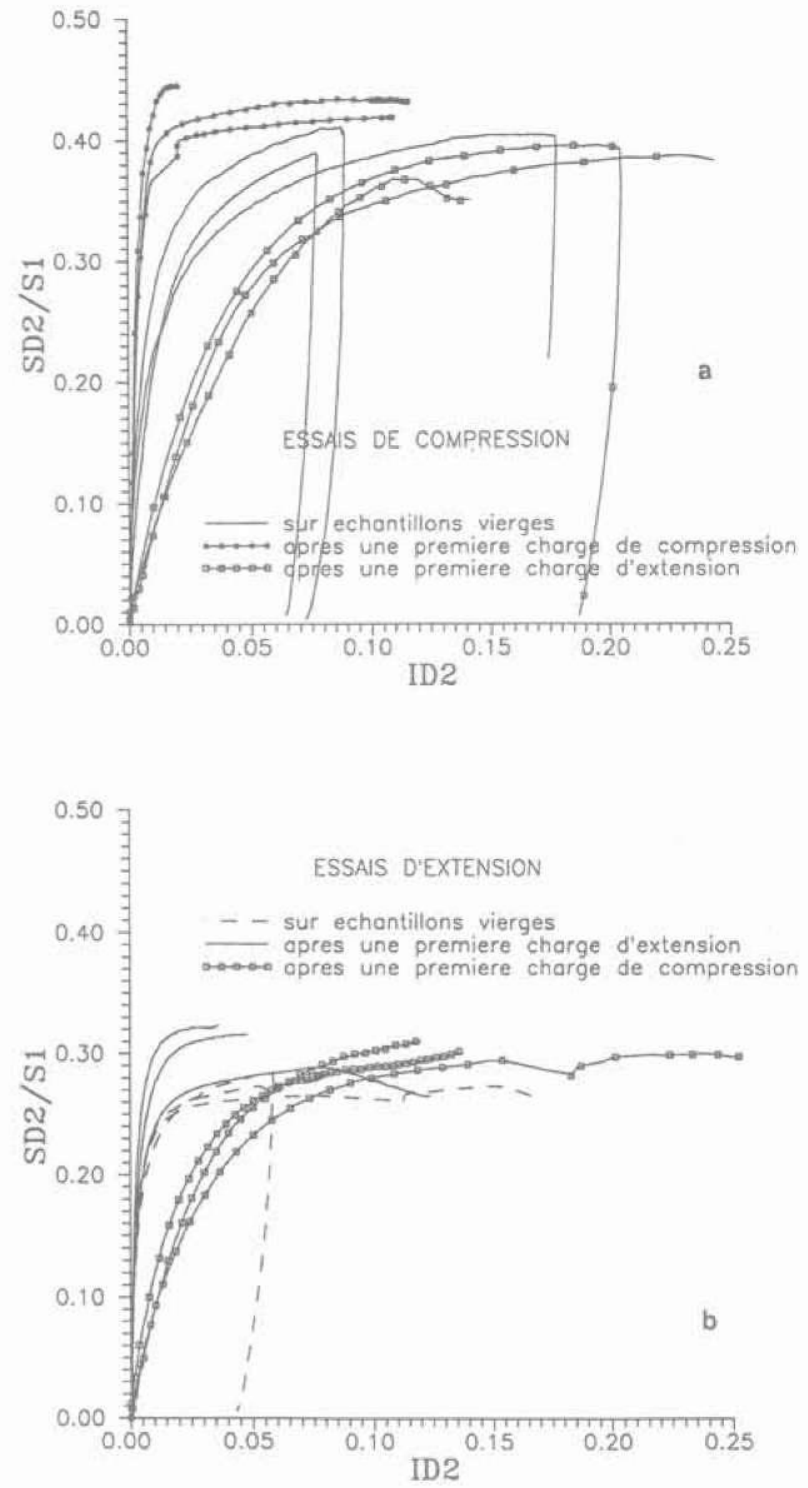

Fig. 16. - Influence de l'anisotropie induite sur les courbes contrainte-déformation (sable dense) : (a) compression, (b) extension.

Fig. 16. - Effects of induced anisotropy on stress-strain curves (dense sand): (a) compression tests, (b) extension tests.

- l'influence de la pression moyenne $\mathrm{p}$ : le compactage initial est plus important quand la pression moyenne augmente, plus faible si elle diminue;

- l'influence de la première charge en relation avec les courbes contrainte-déformation : plus la rigidité de l'échantillon est grande, plus le compactage est faible.

Sur les figures $18 \mathrm{a}-\mathrm{b}-\mathrm{c}$, nous avons regroupé pour chaque type d'échantillon les courbes classiques Q- $\Gamma$. Le point marquant ici est l'évolution du module initial : alors que la pente des courbes parait être la même, quelque soit le chemin de contrainte, pour les échantillons vierges (isotropes), elle est très différente en compression et en extension pour les échantillons anisotropes. Lorsqu'il s'agit d'une "recharge " de compression (ou d'extension) le matériau est très rigide ; s'il s'agit d'une " décharge », la rigidité est faible. Une interprétation qualitative de ce comportement est en- 

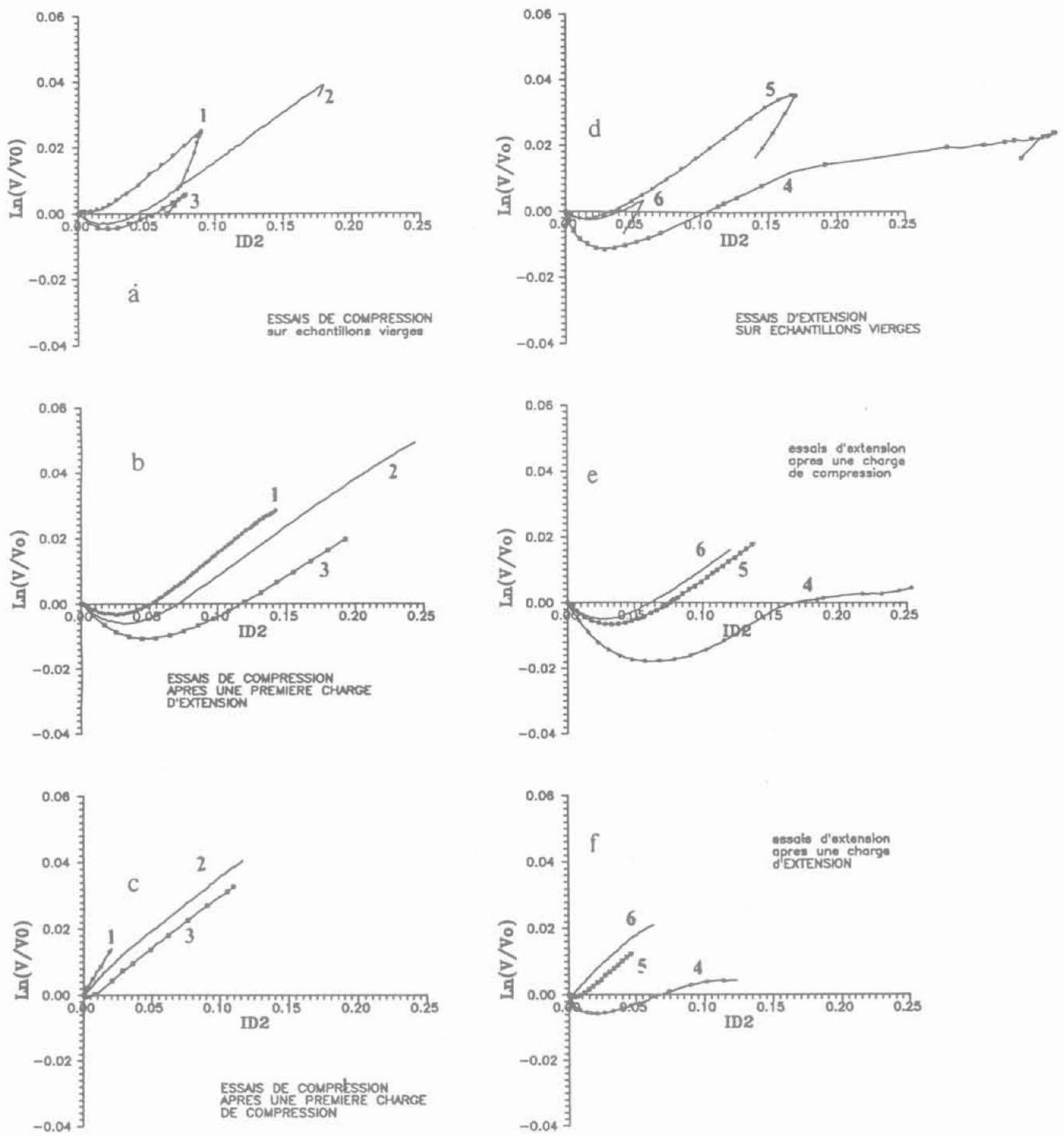

Fig. 17. - Comparaison des courbes de variation de volume pour les 3 types d'échantillons: (a), (b), (c) : essais de compression - (d), (e), (f) : essais d'extension.

Fig. 17. - Comparison of volume change for isotropic and anisotropic samples: (a), (b), (c) compression tests - (d), (e), (f) extension tests.

core une fois possible dans le cadre de l'élasto-plasticité, en imaginant que l'état initial, dans le cas des échantillons anisotropes, appartient à une surface de charge. Comme pour les essais radiaux dans un plan déviatoire et pour avoir l'allure de cette surface de charge, nous avons représenté sur la figure 19 les lignes isodéformations (caractérisées par I2 $=\sqrt{\operatorname{tr}(\epsilon)^{2}}$
= constante) dans le plan $\mathrm{p}-\mathrm{Q}$ de contrainte. Ce type de représentation a été utilisé par GUDEHUS (1980) pour donner une image géométrique des lois incrémentales. Ici encore, à la vue de ces courbes, un écrouissage cinématique paraît nécessaire pour interpréter le comportement du matériau dans le cadre de l'élasto-plasticité. 

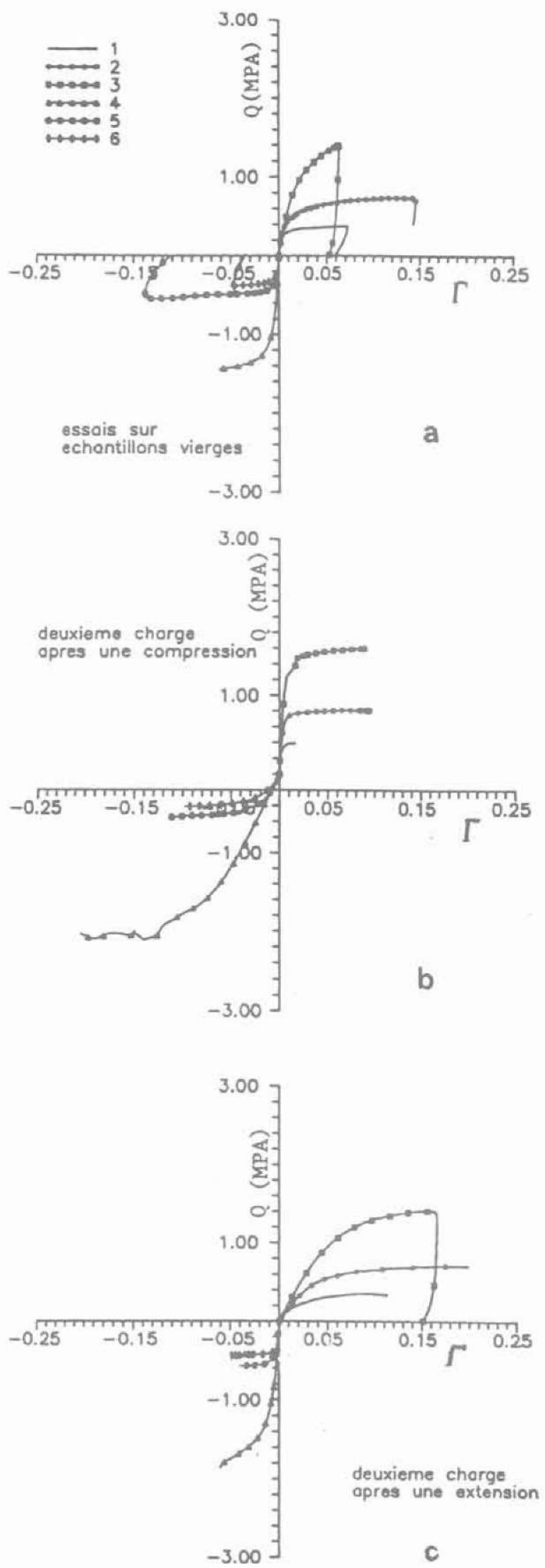

Fig. 18. - Courbes contrainte-déformation $(Q-I)$ : (a) échantillons vierges, (b) échantillons ayant subis une première charge de compression, (c) échantillons ayant subis une première charge d'extension.

Fig. 18, - Stress-strain curves $(Q-\Gamma)$ :

(a) virgin isotropic samples, (b) after a compression test, (c) after an extension test:

\subsection{Chemins axisymétriques $\sigma_{1} / \sigma_{3}=$ constante (sable dense)}

Pour compléter cette étude sur le comportement des sables, nous avons également étudié le comportement de notre matériau sur des chemins radiaux dans le plan ( $\mathrm{p}-\mathrm{Q})$, tels que $\sigma_{1} / \sigma_{3}=$ constante. Ces chemins sont souvent considérés comme les plus réversibles et de ce fait justifient l'hypothèse de surface de charge en forme de cône souvent utilisée dans la modélisation. La prise en compte de l'irréversibilité due à l'augmentation de la pression moyenne est alors obtenue en ajoutant un deuxième mécanisme de déformation plastique (modèle avec «cap $»$ ).

Pour représenter nos résultats expérimentaux et analyser la réponse cinématique du matériau, nous avons choisi d'étudier la direction de l'incrément de déformation totale dє. Dans le cas d'essais axisymétriques, l'incrément de déformation est défini par:

$$
\begin{aligned}
& \mathrm{d} \epsilon_{\mathrm{v}}=\mathrm{d} \epsilon_{1}+2 \cdot \mathrm{d} \epsilon_{3} \\
& \mathrm{~d} \Gamma=\sqrt{2 / 3} \cdot\left(\mathrm{d} \epsilon_{1}-\mathrm{d} \epsilon_{3}\right)
\end{aligned}
$$

avec $\mathrm{p}=\left(\sigma_{1}+2 \cdot \sigma_{3}\right) / 3$ et $\mathrm{Q}=\sqrt{2 / 3} \cdot\left(\sigma_{1}-\sigma_{3}\right)$, cette définition entraîne :

$$
\mathrm{dW}=\sigma_{\mathrm{ij}} \cdot \mathrm{d} \epsilon_{\mathrm{ij}}=\mathrm{p} \cdot \mathrm{d} \epsilon_{\mathrm{v}}+\mathrm{Q} \cdot \mathrm{d} \Gamma
$$

Sur nos figures, la direction de de est représentée par un vecteur de longueur constante. Cette représentation permet plusieurs observations :

- dans le cas où le chemin de contrainte est réversible, ce vecteur doit seulement changer de sens sur les chemins de charge-décharge;

- dans le cas où l'on rencontre une « surface de charge ", l'apparition de déformation plastiques additionnelles peut provoquer une discontinuité sur la direction de $\mathrm{d} \epsilon$.

Les figures 20 présentent les résultats que nous avons obtenus pour l'histoire de chargement suivante (figure 20a) :

- 0--1 : chemin triaxial classique pour atteindre un rapport $\sigma_{1} / \sigma_{3}=4$ (la résistance maximale du matériau correspondant à $\sigma_{1} / \sigma_{3}=4,5$ ).

- 1-2--3=1, puis 3--2--4-5=1: cycles de charge-décharge avec $\sigma_{1} / \sigma_{3}=4$ et $\mathrm{p}_{1}=\mathrm{p}_{3}=\mathrm{p}_{5}$ $=0,4 \mathrm{MPa}, \mathrm{p}_{2}=1,0 \mathrm{MPa}, \mathrm{p}_{4}=1,6 \mathrm{MPa}$.

- le chemin 5 '- -6 est également un chemin radial avec $\sigma_{1} / \sigma_{3}=3$ et $\mathrm{p}_{6}=3,5 \mathrm{MPa}$.

La relation $p-\epsilon_{z}$ pour ce chemin de chargement est représentée sur la figure $21 \mathrm{~b}$. La direction du vecteur incrément de déformation pour différents cycles de charge-décharge est indiquée sur les figures 20c-d-e.

Plusieurs points sont intéressants à noter :

- les cycles de décharge-recharge entre les points 2-3 et 4-5 sont pratiquement réversibles sans boucle d'hystérésis ;

- le chemin 3-2-4 reprend la courbe de première charge lorsque l'on atteint le point d'inversion (point 2). Classiquement on observe alors une forte dimi- 


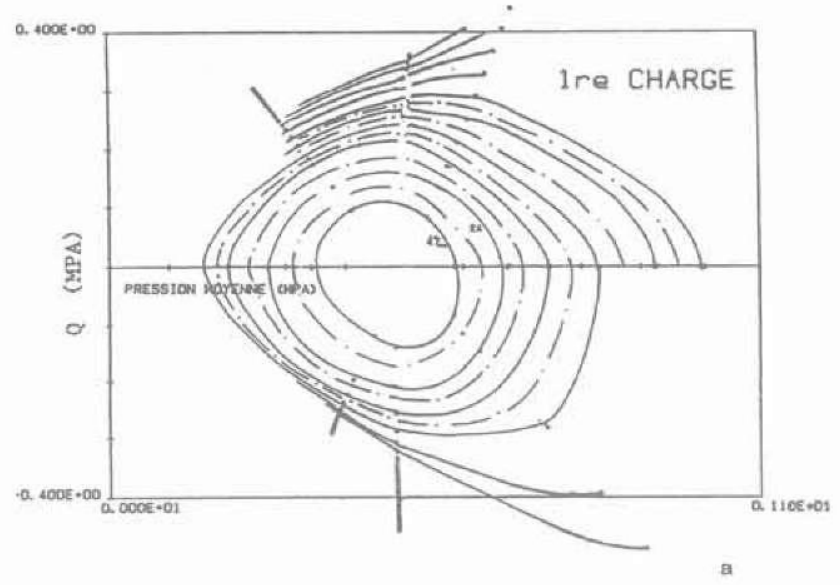

Fig. 19. - Lignes isodéformation ( 12 = constante) dans le plan $p-Q$ : (a) échantillons vierges, (b) échantillons ayant subis une première charge de compression, (c) échantillons ayant subis une première charge d'extension.

Fig. 19. -1 so-strain lines $(12=$ constant) in $p-Q$ plane: (a) virgin isotropic samples, (b) after a compression test, (c) after an extension test.
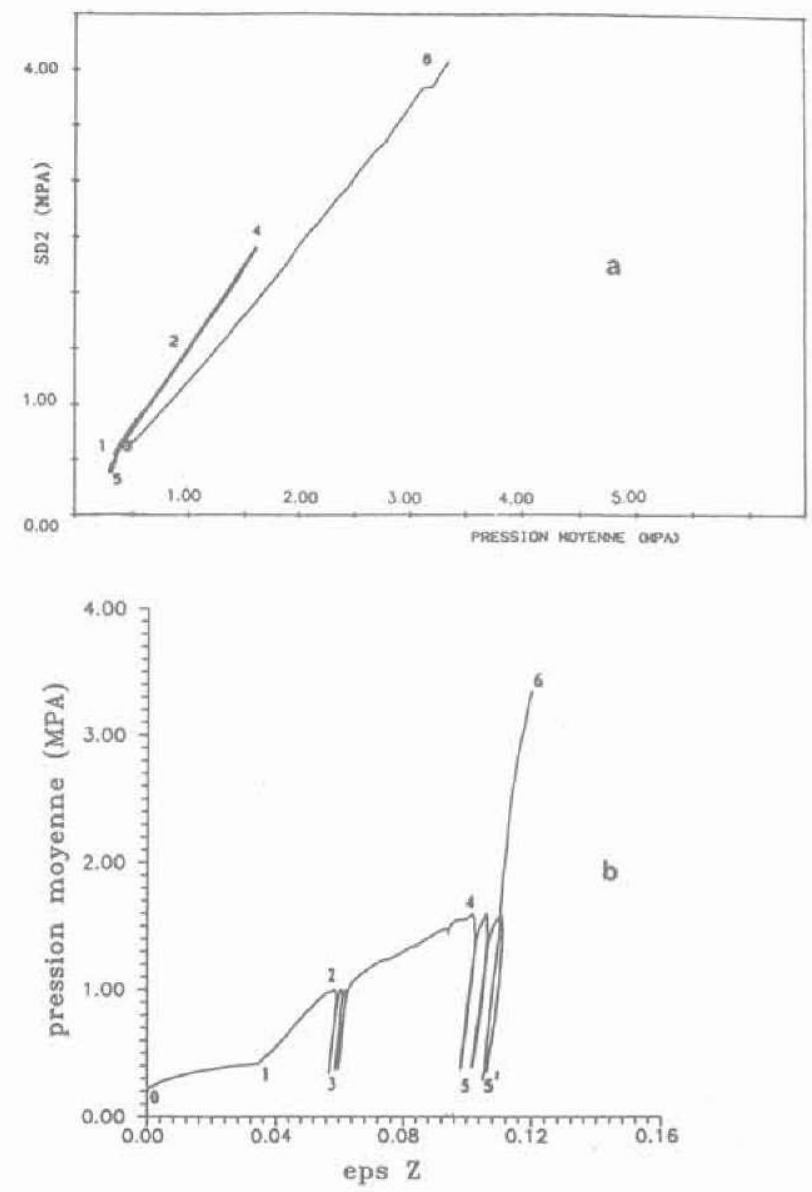

Fig. 20. - Chemins axisymétriques $\sigma_{7} / \sigma_{3}=$ constante: (a) chemin de contrainte dans le plan $p-Q$,

(b) courbe contrainte déformation $p-\epsilon_{\mathrm{Z}}$.

Fig. 20. - Axisymetric test with $\sigma_{7} / \sigma_{3}=$ constant:

(a) stress path in (p-Q) plane, (b) stress-strain curve $p-\epsilon_{2}$.
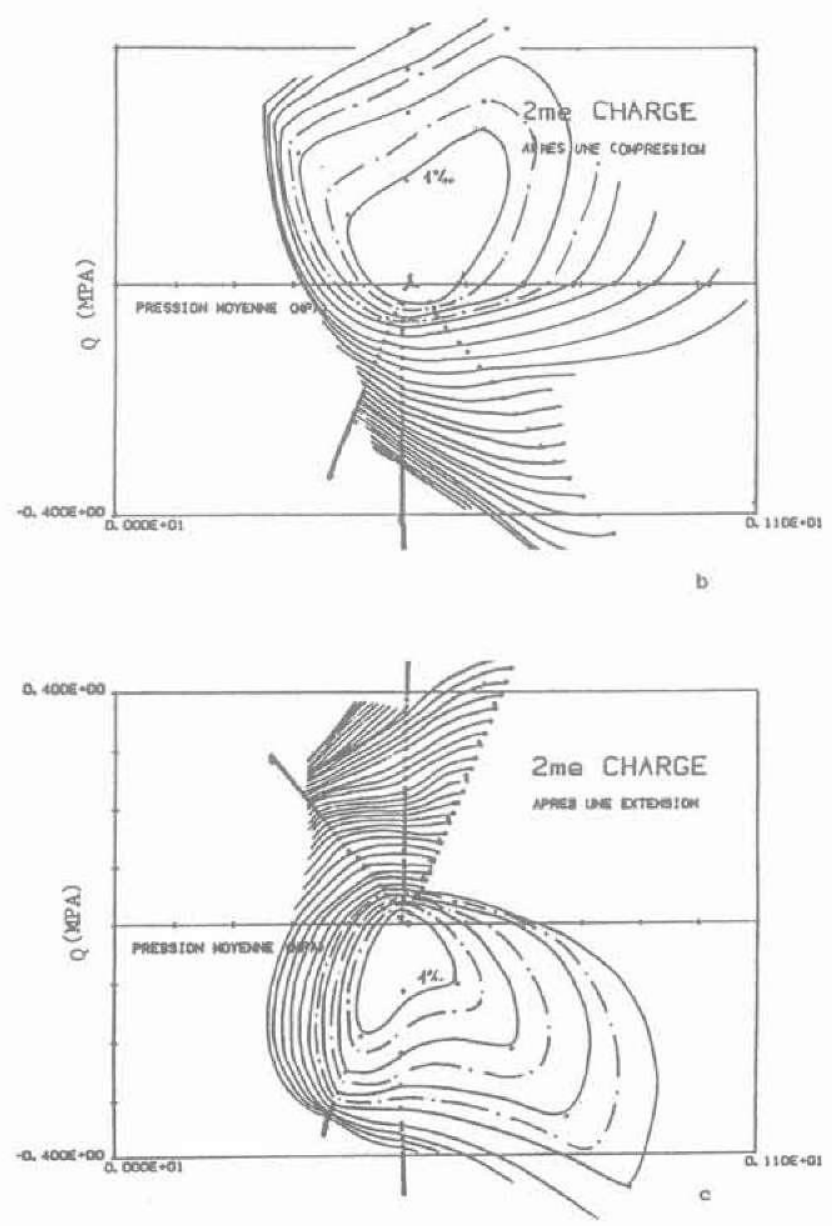

nution de la rigidité du matériau (fig. 20b), mais de plus la direction de l'incrément de déformation subit une discontinuité au passage du point 2 (fig. 20d) ; - on n'observe rien de particulier sur le chemin 5'-6 bien que le niveau de contrainte au point 6 dépasse largement ceux atteints précédemment. On peut cependant noter que la direction de l'incrément de déformation (environ $20^{\circ}$ ) est comparable à celle des chemins quasiment réversibles signalés précédemment, alors que, pour un essai identique $\left(\sigma_{1} / \sigma_{3}=3\right)$ sur un échantillon vierge, cette direction serait comparable à la direction de charge de la figure 20c (environ $\left.80^{\circ}\right)$. L'influence de l'histoire de chargement est donc ici encore très nette.

Si nous faisons encore une fois appel à la théorie de l'élastoplasticité pour interpréter ces résultats nous pouvons faire les remarques suivantes:

- l'inclinaison pratiquement constante du vecteur incrément de déformation sur les chemins $\sigma_{1} / \sigma_{3}=$ constante nous indique que le potentiel plastique est fonction du rapport $\mathrm{q} / \mathrm{p}$ et indépendant de la pression moyenne dans le domaine de contrainte que nous avons envisagé. C'est une hypothèse souvent utilisée (NOVA, WOOD, 1979 ; POOROSHABS, PIETRUSZCZAK, 1985 ; CAMBOU, JAFARI, 1988) ;

- le brusque changement de comportement au passage du point d'inversion peut être interprété par 


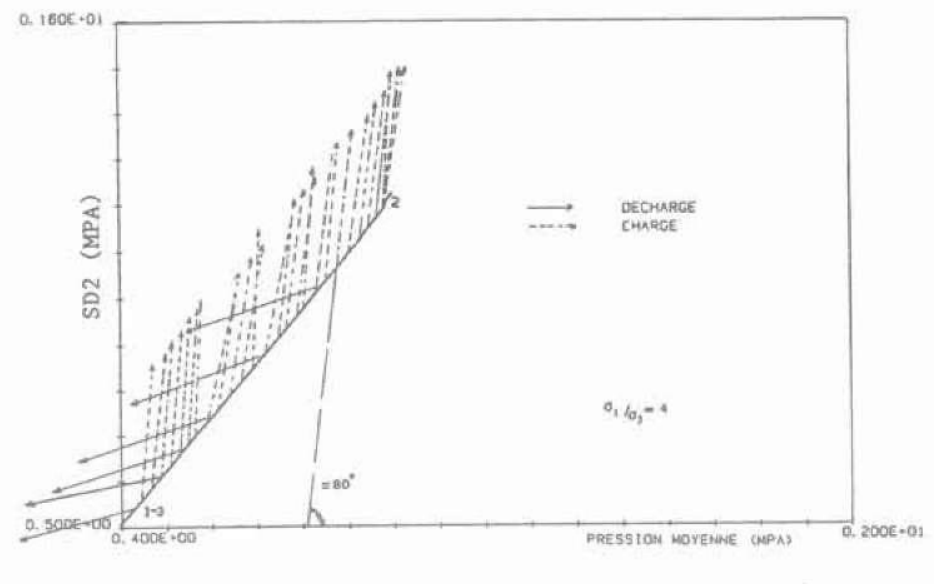

a

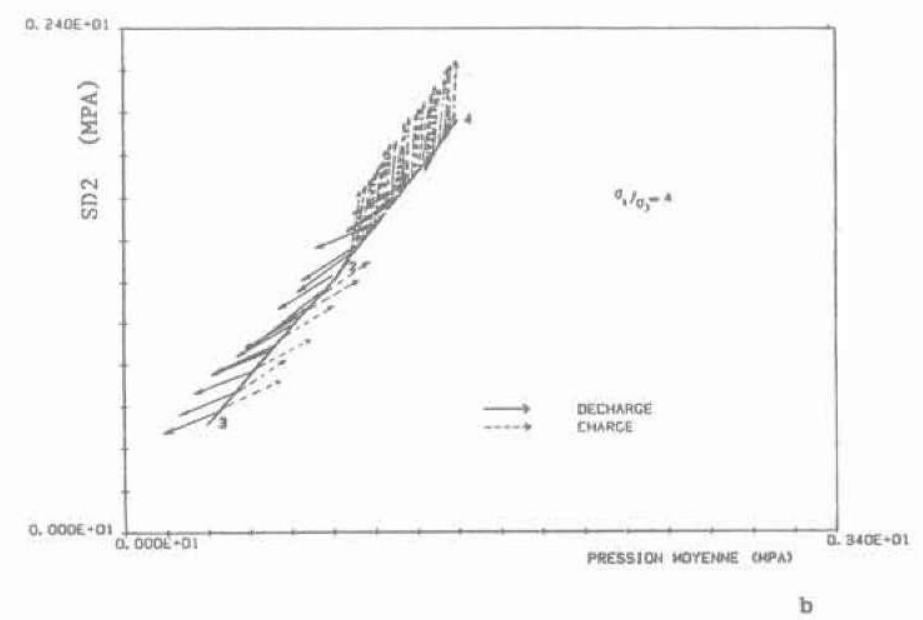

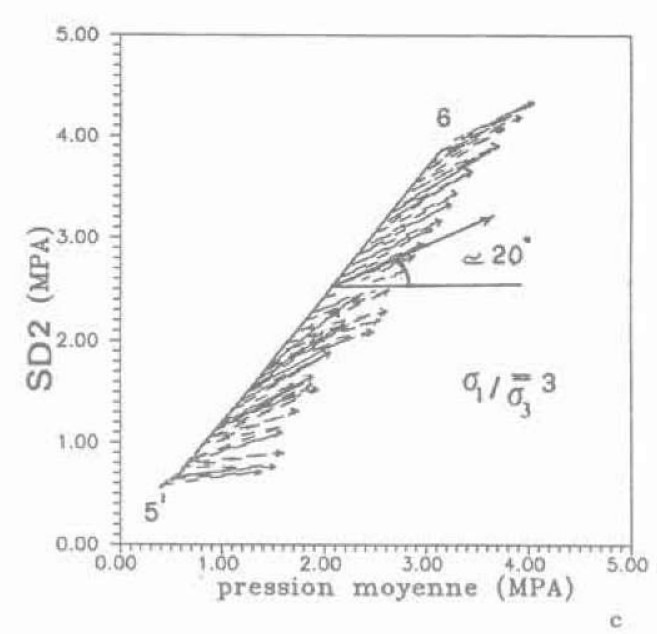

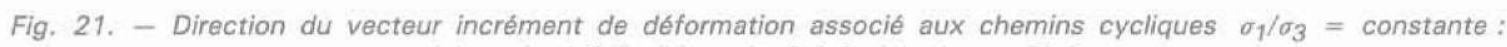
(a) cycle 1-2-3, (b) cycle 3-4-3, (c) charge 5'-6.

Fig. 21. - Inclination of total strain increment associated with cyclic test $\sigma_{1} / \sigma_{3}=$ constant.

l'existence d'une surface de charge de type «cap ». Malheureusement l'identification de cette surface sur le chemin 5'-6 paraît délicate. Des essais complémentaires devront être développés pour éclaircir ce point.

\section{CONCLUSION}

L'étude que nous avons présentée dans cet article nous a montré le rôle important de l'anisotropie créée par une histoire de chargement, pour le sable lâche comme pour le sable dense, pour des chemins de contrainte déviatoires ou axisymétriques. La modification des propriétés mécaniques du matériau concerne sa rigidité et sa compressibilité (variation de volume). D'un point de vue pratique nous avons montré que l'anisotropie pouvait être une cause de liquéfaction, même pour des échantillons denses. Ce fait expérimental ne semble pas avoir été énoncé auparavant.

Nos résultats montrent également que, pour des déformations suffisamment importantes, le comportement plastique, c'est-à-dire la résistance maximale et la loi d'écoulement, est peu modifié. On retrouve ainsi l'idée d'un état "critique » vers lequel évolue la structure du matériau, indépendamment de l'histoire subie précédemment.

Bien qu'un modèle mathématique, fondé sur ces données, ne soit pas encore formulé par les auteurs, nous avons montré que beaucoup des effets observés pouvaient être interprétés dans le cadre de la théorie élastoplastique avec écrouissage isotrope et cinématique. Une modélisation du comportement des géomatériaux dans ce cadre nous paraît donc une voie prometteuse.

\section{REMERCIEMENTS}

La collaboration franco-italienne qui a donné lieu à cet article a été possible grâce au soutien du GRECO Européen sur les géomatériaux.

\section{BIBLIOGRAPHIE}

ARTHUR J.R.F., CHUA K.S., DUNSTAN T. (1977), Induced anisotropy in a sand. Géotechnique 27, 1 , pp. $13-30$. 
ARTHUR J.R.F., CHUA K.S., DUNSTAN T., RODRIGUEZ del C. (1980), Principal stress rotation: a missing parameter. J. Geotech. Eng. Div., ASCE, 106.

CAMBOU B., JAFARI K. (1988), Modèle de com. portement des sols non cohérents. Rev. Franç. de Géotech. 44, pp. 43-55.

CAMBOU B., LANIER J. (1988), Induced anisotropy in cohesionless soil : experiments and modelling. Computers and Geotechnics, 6, pp. 291-311.

FLAVIGNY E., DESRUES J., PALAYER B. (1990), Note technique sur le sable d'Hostun a RF ». Rev. Franç. Géotech. 53, pp. 67-70.

GUDEHUS G. (1980), A comparison of some constitutive laws for soils under radially symmetric loa. ding and unloading. Proc. int. Conf. Num. Meth. Geomech. ed. W. Wittke, Balkema, vol. 4, J. Méca, 19 (2).

LANIER J. (1983), Déformation d'un échantillon de sable sur chemins de contrainte isotrope et triaxiaux de révolution: influence de l'histoire. Rev. Franç. Géotech. 20, pp. 21-29.
NOVA R., WOOD D.M. (1979), A constitutive model for sand in triaxial compression. Int. J. Num. Anal. Meth. Geomech. 3, pp. 255-278.

ODA M. (1972), The mechanism of fabric changes during compressional deformation of sand. Soils and foundations, 12,2 , pp. 1-18.

POOROOSHASB H.B., PIETRUSZCZAK S. (1985), On yielding and flow of sand, a generalized throw surfaces models. Computers and geotechnics, 1, pp. 33-58.

SYMES M.J.P.R., GENS A., HIGHT D.W. (1984), Undrained anisotropy and principal stress rotation in satured sand. Géotechnique 34, pp. 11-27.

YAMADA G., ISHIHARA K. (1979), Anisotropic deformation characteristics of sand under three dimensionnal stress conditions. Soils and Foundations, 19, 2, pp. 79-94.

WONG R.K.S., ARTHUR J.R.F. (1987), Sand sheared by stress with cyclic variation in direction. Geotech. Testing J., 10, pp. 215-226. 